\title{
MERGING DECISION-MAKING UNITS WITH INTERVAL DATA
}

\author{
SAEID GHOBADI*
}

\begin{abstract}
This paper deals with the problem of merging units with interval data. There are two important problems in the merging units. Estimation of the inherited inputs/outputs of the merged unit from merging units is the first problem while the identification of the least and most achievable efficiency targets from the merged unit is the second one. In the imprecise or ambiguous data framework, the inverse DEA concept and linear programming models could be employed to solve the first and second problem, respectively. To identify the required inputs/outputs from merging units, the merged entity is enabled by the proposed method. This provides a predefined efficiency goal. The best and worst attainable efficiency could be determined through the presented models. The developed merging theory is evaluated through a banking sector application.
\end{abstract}

Mathematics Subject Classification. 90C05, 90C29, 90C39, 90C90, 90B50, 47N10.

Received July 22, 2019. Accepted March 6, 2020.

\section{INTRODUCTION}

The data envelopment analysis (DEA) is known as a non-parametric technique for measuring the relative efficiencies of a set of decision-making units (DMUs) with multiple input and output levels. This technique is a powerful mathematical tool that has been employed in different applications like management and economics. In this technique, each unit looks for most desirable set of weights to maximize its efficiency. Therefore, the efficiency of the DMUs could be measured by DEA technique from the optimistic viewpoint. DEA was initially introduced by Charnes et al. [8] and developed from both theoretical and practical aspects by many scholars, see e.g. Cook and Seiford [9], Cooper et al. [11], Emrouznejad et al. [16], Emrouznejad and Yang [15], Ghobadi et al. [27], and Moonesian et al. [40] for some reviews.

In the conventional DEA models, it is assumed that the input and output levels are known exactly. However, this assumption may not be realized in some realistic situations. In other words, the input and output levels in actual problems (such as the efficiency of different bank branches) are often imprecise or ambiguous. Although the stochastic DEA (SDEA) approach has been proposed to deal with imprecise or ambiguous data, due to small sample sizes and specific error distribution assumption, this approach could not be employed for performance evaluation of the DMUs [42]. Therefore, to deal with imprecise or ambiguous data, two main approaches including the fuzzy DEA (FDEA) and the interval DEA (IDEA) have been utilized for performance evaluation of DMUs in the literature. The FDEA and IDEA approaches have been proposed by Sengupta [42] and Cooper et al. [10],

Keywords. Data envelopment analysis (DEA), inverse DEA, merging DMUs, interval data, multiple-objective programming (MOP).

Department of Mathematics, Islamic Azad University, Khomeinishar Barnch, Isfahan, Iran.

*Corresponding author: ghobadi@iaukhsh.ac.ir 
respectively. In Cooper et al. [10], an interval approach based method has been developed by transforming the DEA model into a linear programming (LP) one in the presence of both imprecise and precise data. This paper deals with the IDEA approach based models to achieve their goals. Although, the proposed method could be extended to deal with the FDEA approach, this is not studied in this paper.

Estimation of the lower and upper bounds of the unit's efficiencies is known as an important issue in the interval approach. This issue has been studied by some researchers to present various results, including Despotis and Smirlis [12], Entani et al. [20], Kao [34], and Wang et al. [45]. According to the pessimistic and optimistic viewpoints, an appropriate method has been proposed by Entani et al. [20] to estimate the efficiency and inefficiency intervals for each DMU with imprecise data. Despotis and Smirlis [12] presented a pair of LP models to estimate the upper and lower bounds of efficiency in the presence of interval data. They employed different production technologies to estimate the efficiency score of DMUs that makes their comparison impossible. In fact, using different production possibility sets (PPS) is a drawback of the models proposed by Despotis and Smirlis [12]. To overcome this drawback, these models have been revisited by employing the unique production technology that provides the possibility of measuring the lower and upper bounds of efficiency for each unit [45]. The models proposed by Wang et al. [45] are utilized to achieve the objectives of this paper. Various theoretical and practical aspects of the IDEA approach have been investigated in the literature, including Shokouhi et al. [44], Jahanshahloo et al. [29,31], and Emrouznejad et al. [17,18].

The inverse DEA idea has been initially introduced by Zhang and Cui [51]. In inverse DEA, an efficiency level is considered as a strategic target and the main goal is to estimate the required input and output levels to achieve this pre-determined efficiency level while the goal in the traditional DEA models is to measure the efficiency score of a DMU with known input and output levels. According to the DEA literature, the various theoretical and practical aspects of the inverse DEA have been studied by some researchers, including sensitivity analysis [30], setting revenue target [13,37], resource allocation [19,22,25,28], investment analysis [32,48], under inter-temporal dependence of data [24,33], with imprecise data [23,26], and firms restructuring [3].

In the business world, several companies are usually merged to generate a new company with superior performance. Generally, a merger is formed when at least two DMUs combine their activities to generate a new merged unit with better performance. Moreover, these companies acquire other businesses to obtain novel technologies or decrease competition. These companies could be converted to more effective, beneficial and strong companies by means of the mentioned mergers and acquisitions (M\&As). According to Motis [41] studies, the proposed motives for M\&As are based on the shareholder gains (such as efficiency and synergy) or based on the managerial gains (such as empire building or managerial discretion). In this paper, obtaining the maximum benefit by saving inputs or maximizing outputs is the main motive for M\&A. According to the consummated or proposed acquisitions, Weber and Dholakia [47] provided a method to identifying, valuing, and prioritizing opportunities for marketing synergy. This method can help improve the chances for more successful M\&As. The performance of the effects of M\&As is examined through a banking empirical application [6]. The research results show that the productivity has not been fully improved due to inefficient mergers. According to the M\&A literature, the effects of M\&A have been studied in many theoretical and applied publications, including telecommunications [38], agriculture [7], healthcare [35], railway [5] and banking [39,46].

The DEA technique is a powerful mathematical tool that can be used to evaluate of the mergers performance in different field. Estimation of the inherited inputs and outputs from merging DMUs is an important topic. The inverse DEA has been firstly employed in the merging DMUs problem to identify the inherited input/output levels from merging units to achieve the pre-defined target level by Gattoufi et al. [21]. Zeinodin and Ghobadi [49] provided a new approach to answer the problem of merging units using Pareto solutions of linear multipleobjective programming (MOLP) problems. An important advantage of the proposed approach, compared to the inverse DEA models proposed in Gattoufi et al. [21], decreases the number of the variables of the model strongly. This leads to reduced computational complexity. According to the inverse DEA and goal programming, Amin et al. [4] have proposed a new model for target setting of a merger. This study is an important practical method that allows managers to incorporate their priority in target setting of a merger to reach the maximum benefit either by saving inputs or maximizing outputs. Amin and Oukil [2] presented the inverse DEA based method 
for flexible target setting of a merger. The presented method allows the decision maker to prioritize specific input in the target settings. A two-phase method suggested to estimate of the potential merger efficiency of a hypothetical unit from the cost viewpoint by Shi et al. [43]. Also, a new method is proposed for analyzing the potential overall gains from mergers into technical, size, and harmony effects by Li et al. [36]. According to this study, it was concluded that the technical and harmony effects would work favor mergers, while the size effect would work against most mergers. According to the DEA literature, the inverse DEA has been adopted to solve the problem of merging DMUs with negative data and under inter-temporal dependence data by Amin and Al-Muharrami [1] and Zenodin and Ghobadi [50], respectively. However, the proposed models in these studies could not solve the merging DMUs problem in the presence of interval data. Therefore, the following questions in the IDEA framework are raised:

Question 1. If a subset of DMUs is required for merging and creating a new DMU with a definite input/output level and a predetermined efficiency target, what should be the output/input level of the merged DMU?

Question 2. What are the least and most achievable efficiency targets through a given merging?

To answer Question 1, an inverse DEA method is proposed. In other words, novel inverse DEA models are provided to achieve a pre-defined efficiency target of a merger with interval data. The proposed models enables the merged DMU to estimate the required values of inputs/outputs from merging DMUs to achieve a prespecified efficiency level. Sufficient conditions for estimating the upper and lower bounds of the input/output levels of the merged DMU are established through the Pareto and weak Pareto solutions of multiple-objective linear programming problems. The paper also provides insightful models to answer Question 2. In other words, a method proposed to determine the least and most achievable efficiency values through a given merging. Although the proposed method could be utilized in several applications, its performance is investigated through a banking sector example.

The current paper proceeds as follows: Section 2 provides some preliminaries from multiple-objective optimization and DEA with interval data. The main results of the paper are presented in Sections 3 and 4. In Section 3, identification of the inherited input/output levels are dealt with. In Section 4, a method is proposed for determining the minimum and maximum attainable values of the upper and lower performance through a merger. In Section 5, a banking sector application is presented to demonstrate the developed merging theory. Section 6 gives a brief conclusion and future research directions.

\section{Preliminaries}

\subsection{Multiple-objective programming}

A general multiple-objective programming (MOP) problem is as

$$
\begin{array}{ll}
\max & F(x) \\
\text { s.t. } & X=\left\{x \in \mathbb{R}^{m}: G(x) \leq 0\right\},
\end{array}
$$

where $F(x)=\left(f_{1}(x), f_{2}(x), \ldots, f_{n}(x)\right), \quad G(x)=\left(g_{1}(x), g_{2}(x), \ldots, g_{p}(x)\right)$.

$x \in X$ is called a feasible solution to MOP (2.1). $f_{j}$ s are the objective functions of this MOP. There is usually no feasible solution that simultaneously maximizes all objective functions. Therefore, (weak) Pareto solutions are defined instead of optimal solutions.

Definition 2.1 (Ehrgott [14]). Let $\bar{x}$ be a feasible solution to MOP (2.1).

(i) If there does not exist $x \in X$ such that

$$
\begin{aligned}
& f_{j}(\bar{x}) \leq f_{j}(x) \text { for each } j=1,2, \ldots, n, \\
& f_{j}(\bar{x})<f_{j}(x) \text { for some } j=1,2, \ldots, n,
\end{aligned}
$$

then $\bar{x}$ is called a Pareto solution to MOP (2.1). 
(ii) If there does not exist $x \in X$ such that

$$
f_{j}(\bar{x})<f_{j}(x) \text { for each } j=1,2, \ldots, n,
$$

then $\bar{x}$ is called a weak Pareto solution to MOP (2.1).

\subsection{DEA with interval data}

Suppose that there is a set of $n$ DMUs, $\left\{\mathrm{DMU}_{j}: j \in J=\{1,2, \ldots, n\}\right\}$, to be evaluated. Assume that the $\mathrm{DMU}_{j}$ consumes $\mathrm{m}$ inputs $x_{i j}$ in order to produce s outputs $y_{r j}$, for all $i=1,2, \ldots, m, r=1,2, \ldots, s$, and $j=1,2, \ldots, n$. Unlike the conventional DEA, suppose that the input and output levels of $\mathrm{DMU}_{j}$ are not known exactly. In other words, the true input and output levels of $\mathrm{DMU}_{j}$ are known to lie within bounded intervals, i.e. $x_{i j}=\left[x_{i j}^{l}, x_{i j}^{u}\right]$ and $y_{r j}=\left[y_{r j}^{l}, y_{r j}^{u}\right]$, where the lower and upper bounds of intervals given as constants and assumed positive. Also, $x_{i j}^{l} \leq x_{i j}^{u}$ and $y_{r j}^{l} \leq y_{r j}^{u}$. In this case, the efficiency score of $\mathrm{DMU}_{o}, o \in J$ can be an interval as $\theta_{o}=\left[\theta_{o}^{l}, \theta_{o}^{u}\right]$, where $\theta_{o}^{l}$ and $\theta_{o}^{u}$ are the lower and upper bounds of the efficiency interval $\mathrm{DMU}_{o}$, respectively. According to the pessimistic and optimistic viewpoints, Despotis and Smirlis [12] presented a pair of LP models to estimate the upper and lower bounds of efficiency. In these models, variable production possibility set (PPS) is employed to measure the efficiencies of DMUs. Accordingly, their comparison becomes impossible. To measure the efficiencies of all DMUs, the models proposed by Despotis and Smirlis [12] have been modified by Wang et al. [45] by using a fixed and unified PPS. In order to the unit assessment of $\mathrm{DMU}_{o}$, the envelopment form of these DEA models under variable returns to scale (VRS) assumption are formulated as follows:

$$
\begin{aligned}
\theta_{o}^{l}= & \min \theta \\
\text { s.t. } & \sum_{j=1}^{n} \lambda_{j} x_{i j}^{l} \leq \theta x_{i o}^{u}, \quad i=1,2, \ldots, m, \\
& \sum_{j=1}^{n} \lambda_{j} y_{r j}^{u} \geq y_{r o}^{l}, \quad r=1,2, \ldots, s, \\
& \sum_{j=1}^{n} \lambda_{j}=1, \\
& \lambda_{j} \geq 0, \\
\theta_{o}^{u}= & \min \theta \quad j=1,2, \ldots, n . \\
\text { s.t. } & \sum_{j=1}^{n} \lambda_{j} x_{i j}^{l} \leq \theta x_{i o}^{l}, \quad i=1,2, \ldots, m, \\
& \sum_{j=1}^{n} \lambda_{j} y_{r j}^{u} \geq y_{r o}^{u}, \quad r=1,2, \ldots, s, \\
& \sum_{j=1}^{n} \lambda_{j}=1, \\
& \lambda_{j} \geq 0, \quad j=1,2, \ldots, n .
\end{aligned}
$$

In models $(2.2)$ and $(2.3),(\lambda, \theta)$ is the variables vector. It is not difficult to see that $\theta_{o}^{l} \leq \theta_{o}^{u} \leq 1$. These models are in the input-oriented. Wang et al. [45] defined the efficient unit as follows:

Definition 2.2. $\mathrm{DMU}_{o}$ is called input-oriented efficient if and only if $\theta_{o}^{u}=1$; otherwise, it is inefficient. 
The output-oriented version of the models (2.2) and (2.3) are as follows:

$$
\begin{aligned}
& \varphi_{o}^{u}=\max \varphi \\
& \text { s.t. } \quad \sum_{j=1}^{n} \lambda_{j} x_{i j}^{l} \leq x_{i o}^{u}, \quad i=1,2, \ldots, m, \\
& \sum_{j=1}^{n} \lambda_{j} y_{r j}^{u} \geq \varphi y_{r o}^{l}, \quad r=1,2, \ldots, s, \\
& \sum_{j=1}^{n} \lambda_{j}=1, \\
& \lambda_{j} \geq 0, \quad j=1,2, \ldots, n . \\
& \varphi_{o}^{l}=\max \varphi \\
& \text { s.t. } \quad \sum_{j=1}^{n} \lambda_{j} x_{i j}^{l} \leq x_{i o}^{l}, \quad i=1,2, \ldots, m, \\
& \sum_{j=1}^{n} \lambda_{j} y_{r j}^{u} \geq \varphi y_{r o}^{u}, \quad r=1,2, \ldots, s \\
& \sum_{j=1}^{n} \lambda_{j}=1 \text {, } \\
& \lambda_{j} \geq 0, \quad j=1,2, \ldots, n .
\end{aligned}
$$

$(\lambda, \varphi)$ is the variables vector of the models (2.4) and (2.5). Here, $\varphi_{o}^{u}$ and $\varphi_{o}^{l}$ are called upper and lower bounds of the efficiency scores of $\mathrm{DMU}_{o}$, respectively. It is not difficult to see that $1 \leq \varphi_{o}^{l} \leq \varphi_{o}^{u}$. Therefore, $\varphi_{o}=\left[\varphi_{o}^{l}, \varphi_{o}^{u}\right]$ can be considered as the efficiency interval of $\mathrm{DMU}_{o}$.

Definition 2.3. $\mathrm{DMU}_{o}$ is called output-oriented efficient if and only if $\varphi_{o}^{l}=1$; otherwise, it is inefficient.

\section{Merging DMUs in the PRESEnCE of INTERVAl DATA}

According to the M\&A literature [41], the proposed motives for M\&As are based on the shareholder gains (such as efficiency and synergy) or based on the managerial gains (such as empire building or managerial discretion). According to the DEA literature, the methods for modelling a merger based on the inverse DEA concept are proposed to achieve the most benefits either by maximizing outputs or saving inputs $[1,21,50]$. However, the proposed methods in all of the mentioned studies fail for merging DMUs with interval data. This could be due to the limitations of the basic DEA models used in their modeling. In this section based on the achieve approach to the maximum benefit, we propose a method for treating the interval data in the problem of merging DMUs.

Assume that there is a set of selected units to merge such as $\left\{\mathrm{DMU}_{j}, j \in \Lambda \subset J\right\}$. In fact, $\Lambda$ is the set of selected units to create synergy through merging and generate a new unit to achieve given efficiency targets that is shown with $\mathrm{DMU}_{q}$. It is clear that the set of selected units for merge removed after merge. Moreover, $\Pi=J-\Lambda$ is the set of units that have not participated in merger. According to the achieve approach to the maximum benefit in the process of merging units, it is assumed that $\mathrm{DMU}_{q}$ keeps the amount of input/output levels of set of the merging units and looks for the maximum/minimum amount of the inherited output/input levels to achieve given efficiency target.

Sections 3.1 and 3.2 demonstrated that identification of the inherited input and output levels could be achieved through the merged DMU, respectively. 


\subsection{Estimation of input}

Suppose that $\mathrm{DMU}_{q}$ keeps the amount of outputs of set of the merging DMUs and looks for the minimum amount of the inherited sources of these DMUs in order to reach the pre-defined target level, $\bar{\theta}_{q}=\left[\bar{\theta}_{q}^{l}, \bar{\theta}_{q}^{u}\right]$. Therefore, the output levels of merged $\operatorname{DMU}\left(\mathrm{DMU}_{q}\right)$ is $y_{r q}=\left[y_{r q}^{l}, y_{r q}^{u}\right]$, where $y_{r q}^{l}=\sum_{j \in \Lambda} y_{r j}^{l}$ and $y_{r q}^{u}=\sum_{j \in \Lambda} y_{r j}^{u}$ for all $r \in O=\{1,2, \ldots, s\}$; are the lower and upper limit of the inherited output levels of the merging DMUs, respectively. We need to estimate the inputs $x_{i q}=\left[x_{i q}^{l}, x_{i q}^{u}\right]$ provided that the interval efficiency of $\mathrm{DMU}_{q}$ is $\bar{\theta}_{q}=\left[\bar{\theta}_{q}^{l}, \bar{\theta}_{q}^{u}\right]$. In fact,

$$
x_{i q}=\left[x_{i q}^{l}, x_{i q}^{u}\right]=\sum_{j \in \Lambda} \alpha_{i j}=\sum_{j \in \Lambda}\left[\alpha_{i j}^{l}, \alpha_{i j}^{u}\right]=\left[\sum_{j \in \Lambda} \alpha_{i j}^{l}, \sum_{j \in \Lambda} \alpha_{i j}^{u}\right],
$$

where, $\alpha_{i j}=\left[\alpha_{i j}^{l}, \alpha_{i j}^{u}\right]$ is the inherited input level from merging units of $\mathrm{DMU}_{j}$, for all $i \in I=\{1,2, \ldots, m\}$ and $j \in \Lambda$.

In the problem of merging units, if the identification of the maximum receivable merger gains is the main target of the merger, then these gains could be calculated by minimizing the sum of the inherited input levels provided that the there is no priority in saving various inputs. In other words, the corresponding model can be a single objective. However, if there is various objectives for a merger, then the relevant model must be a MOP. In this paper, unlike the approach of Gattoufi et al. [21], we assume that the decision maker seeks various objectives. Therefore, to estimate of the lower limit of the input levels of $\mathrm{DMU}_{q}$ to achieve the upper desired given efficiency target $\bar{\theta}_{q}^{u}$, the following MOP model is proposed:

$$
\begin{aligned}
& \min \left(\alpha_{i j}^{l} ; \forall i \in I, \forall j \in \Lambda\right) \\
& \text { s.t. } \quad \sum_{j \in \Pi} \lambda_{j} x_{i j}^{l}+\left(\sum_{j \in \Lambda} \alpha_{i j}^{l}\right) \lambda_{q} \leq \bar{\theta}_{q}^{u} \sum_{j \in \Lambda} \alpha_{i j}^{l}, \quad \forall i \in I \text {, } \\
& \sum_{j \in \Pi} \lambda_{j} y_{r j}^{u}+y_{r q}^{u} \lambda_{q} \geq y_{r q}^{u}, \quad \forall r \in O, \\
& \sum_{j \in \Pi} \lambda_{j}+\lambda_{q}=1, \\
& 0 \leq \alpha_{i j}^{l} \leq x_{i j}^{l}, \\
& \lambda_{j} \geq 0,
\end{aligned}
$$

In model (3.1), $\left(\lambda_{j} ; j \in \Pi \cup\{q\}, \alpha_{i j}^{l} ; \forall i \in I, \forall j \in J\right)$ is the variables vector. In the above model, the expected efficiency upper bound for $\mathrm{DMU}_{q}$ is denoted by $\bar{\theta}_{q}^{u}$. The goals of the model (3.1) guarantees that the inputs inherited by the merged unit from the participating units in the merger are minimized, in order to achieve the desired efficiency target $\bar{\theta}_{q}^{u}$. In other words, the main motive for M\&A is to reach the maximum benefit by saving inputs in the present section. The following theorem shows how the above MOP can be linearized.

Theorem 3.1. If $D M U_{q}$ is within the current PPS, then model (3.1) can be converted to the following MOLP problem:

$$
\begin{array}{lll}
\min & \left(\alpha_{i j}^{l} ; \forall i \in I, \forall j \in \Lambda\right) \\
\text { s.t. } & \sum_{j \in \Pi} \lambda_{j} x_{i j}^{l} \leq \bar{\theta}_{q}^{u} \sum_{j \in \Lambda} \alpha_{i j}^{l}, \quad \forall i \in I, \\
& \sum_{j \in \Pi} \lambda_{j} y_{r j}^{u} \geq y_{r q}^{u}, \quad \forall r \in O,
\end{array}
$$




$$
\begin{array}{ll}
\sum_{j \in \Pi} \lambda_{j}=1, & \\
0 \leq \alpha_{i j}^{l} \leq x_{i j}^{l}, & \forall i \in I, \forall j \in \Lambda, \\
\lambda_{j} \geq 0, & \forall j \in \Pi .
\end{array}
$$

Proof. If $\mathrm{DMU}_{q}$ is within the current PPS, then $\mathrm{DMU}_{q}$ is interior or upon frontier of the current PPS. If $\mathrm{DMU}_{q}$ is interior of the current PPS, then the generated new unit $\left(\mathrm{DMU}_{q}\right)$ can be stated by a convex combination of some units that have not participated in merger. Therefore, in each Pareto solution of model (3.1), we have $\lambda_{q}^{*}=0$. Accordingly, model (3.1) can be converted to MOLP (3.2). Also, if $\mathrm{DMU}_{q}$ is upon frontier of the current PPS, it can be presented in terms of the other efficient DMUs, and therefore in this case, we can still suppose that $\lambda_{q}^{*}=0$ in optimality. Note that in this case, considering $\lambda_{q}^{*}=0$ means ignoring only one optimal solution from the set of optimal solutions to problem (3.1). Hence, the MOP model (3.1) will be simplified to the same MOLP (3.2). This completes the proof of theorem.

In this paper, according to the above theorem, we limit our development to the case of the consolidation where the merged unit (DMUq) is within the current PPS. Therefore, without consideration this assumption, the topic discussed in this paper can be worth studying as well, though we do not pursue it in the present study.

According to the following theorem, the input-oriented model (3.2) could be utilized to determine the lower limit of the $\mathrm{DMU}_{q}$ input levels.

Theorem 3.2. If the following assumptions hold:

(i) The merged unit (DMUq) is within the current PPS;

(ii) $\Delta^{l}=\left(\lambda^{*}, \alpha_{i j}^{l *}: \forall i \in I, \forall j \in \Lambda\right)$ is a Pareto solution to model (3.2);

(iii) $x_{i q}^{l}=\sum_{j \in \Lambda} \alpha_{i j}^{l *}$ for each $i \in I$.

Then, the obtained efficiency upper bound of $D M U_{q}$ is equal to $\bar{\theta}_{q}^{u}$.

Proof. See Appendix A.

Now, to estimate of the upper limit of the input levels of $\mathrm{DMU}_{q}$ to achieve the lower desired given efficiency target $\bar{\theta}_{q}^{l}$, the following MOLP model is proposed:

$$
\begin{aligned}
& \min \left(\alpha_{i j}^{u} ; \forall i \in I, \forall j \in \Lambda\right) \\
& \text { s.t. } \quad \sum_{j \in \Pi} \lambda_{j} x_{i j}^{l}+\left(\sum_{j \in \Lambda} \bar{\alpha}_{i j}^{l}\right) \lambda_{q} \leq \bar{\theta}_{q}^{l} \sum_{j \in \Lambda} \alpha_{i j}^{u}, \quad \forall i \in I, \\
& \sum_{j \in \Pi} \lambda_{j} y_{r j}^{u}+y_{r q}^{u} \lambda_{q} \geq y_{r q}^{l}, \quad \forall r \in O, \\
& \sum_{j \in \Pi} \lambda_{j}+\lambda_{q}=1 \\
& \bar{\alpha}_{i j}^{l} \leq \alpha_{i j}^{u} \leq x_{i j}^{u}, \\
& \forall i \in I, \forall j \in \Lambda \text {, } \\
& \lambda_{j} \geq 0 \text {, }
\end{aligned}
$$

In the above Model, $\left(\lambda_{j} ; j \in \Pi \cup\{q\}, \alpha_{i j}^{u} ; \forall i \in I, \forall j \in \Lambda\right)$ is the variables vector. In this model, the expected efficiency upper bound for $\mathrm{DMU}_{q}$ is denoted by $\bar{\theta}_{q}^{l}$. Also, $\left(\bar{\alpha}_{i j}^{l} ; \forall j \in \Lambda, \forall i \in I\right)$ is a Pareto solution of model (3.2). Theorem 3.3 shows how the above MOP can be converted to the MOLP (3.4). 
Theorem 3.3. If $D M U_{q}$ is within the current PPS, then model (3.3) can be converted to the following MOLP problem:

$$
\begin{aligned}
& \min \left(\alpha_{i j}^{u} ; \forall i \in I, \forall j \in \Lambda\right) \\
& \text { s.t. } \quad \sum_{j \in \Pi} \lambda_{j} x_{i j}^{l} \leq \bar{\theta}_{q}^{l} \sum_{j \in \Lambda} \alpha_{i j}^{u}, \quad \forall i \in I, \\
& \sum_{j \in \Pi} \lambda_{j} y_{r j}^{u} \geq y_{r q}^{l}, \quad \forall r \in O, \\
& \sum_{j \in \Pi} \lambda_{j}=1 \text {, } \\
& \bar{\alpha}_{i j}^{l} \leq \alpha_{i j}^{u} \leq x_{i j}^{u}, \quad \forall i \in I, \forall j \in \Lambda, \\
& \lambda_{j} \geq 0, \quad \forall j \in \Pi \text {. }
\end{aligned}
$$

Proof. The proof is similar to the proof of Theorem 3.1. Note that the best situation of $\mathrm{DMU}_{q}$ $\left(x_{i q}^{l}=\sum_{j \in \Lambda} \bar{\alpha}_{i j}^{l}, y_{r q}^{u}\right)$ could be described by a convex combination of the best situation of units that have not participated in merger.

Theorem 3.4 shows how MOLP (3.4) could be used to estimate of the upper limit of the $\mathrm{DMU}_{q}$ input levels.

Theorem 3.4. If the following assumptions hold:

(i) The merged unit (DMUq) is within the current PPS;

(ii) $\Delta^{u}=\left(\lambda^{*}, \alpha_{i j}^{u *}: \forall i \in I, \forall j \in \Lambda\right)$ is a Pareto solution to MOLP (3.4) in which $\alpha_{i j}^{u *} \neq \bar{\alpha}_{i j}^{l}$ for some $i \in I$ and $j \in \Lambda$;

(iii) $x_{i q}^{u}=\sum_{j \in \Lambda} \alpha_{i j}^{u *}$ for each $i \in I$.

Then, the obtained efficiency lower bound of $\mathrm{DMU}_{q}$ is equal to $\bar{\theta}_{q}^{l}$.

Proof. See Appendix B.

\subsection{Estimation of output}

In this section, a method is proposed for the identification of the inherited output levels from merging DMUs to attain a pre-defined efficiency target when the data are considered to lie within bounded intervals.

In order to, suppose that $\mathrm{DMU}_{q}$ keeps the amount of inputs of set of the merging DMUs and looks for the maximum amount of the inherited outputs of these DMUs to reach the pre-defined target level, $\bar{\varphi}_{q}=\left[\bar{\varphi}_{q}^{l}, \bar{\varphi}_{q}^{u}\right]$. Therefore, the input levels of merged DMU $\left(\mathrm{DMU}_{q}\right)$ is $x_{i q}=\left[x_{i q}^{l}, x_{i q}^{u}\right]$, where $x_{i q}^{l}=\sum_{j \in \Lambda} x_{i j}^{l}$ and $x_{i q}^{u}=\sum_{j \in \Lambda} x_{i j}^{u}$ for all $i \in I$; are the lower and upper limit of the inherited input levels of the merging DMUs, respectively. We need to estimate the outputs $y_{r q}=\left[y_{r q}^{l}, y_{r q}^{u}\right]$ provided that the efficiency interval of $\mathrm{DMU}_{q}$ is $\bar{\varphi}_{q}=\left[\bar{\varphi}_{q}^{l}, \bar{\varphi}_{q}^{u}\right]$. In fact,

$$
y_{r q}=\left[y_{r q}^{l}, y_{r q}^{u}\right]=\sum_{j \in \Lambda} \beta_{r j}=\sum_{j \in \Lambda}\left[\beta_{r j}^{l}, \beta_{r j}^{u}\right]=\left[\sum_{j \in \Lambda} \beta_{r j}^{l}, \sum_{j \in \Lambda} \beta_{r j}^{u}\right],
$$

where, $\beta_{r j}=\left[\beta_{r j}^{l}, \beta_{r j}^{u}\right]$ is the inherited output levels from merging units of $\mathrm{DMU}_{j}$, for all $r=1,2, \ldots, s$ and $j \in \Lambda$. 
To estimate of the upper limit of the output levels of $\mathrm{DMU}_{q}$ to achieve the lower desired given efficiency target $\bar{\varphi}_{q}^{l}$, the following MOP model is proposed:

$$
\begin{array}{lll}
\max & \left(\beta_{r j}^{u} ; \forall r \in O, \forall j \in \Lambda\right) & \\
\text { s.t. } & \sum_{j \in \Pi} \lambda_{j} x_{i j}^{l}+x_{i q}^{l} \lambda_{q} \leq x_{i q}^{l}, & \forall i \in I, \\
& \sum_{j \in \Pi} \lambda_{j} y_{r j}^{u}+\left(\sum_{j \in \Lambda} \beta_{r j}^{u}\right) \lambda_{q} \geq \bar{\varphi}_{q}^{l} \sum_{j \in \Lambda} \beta_{r j}^{u}, & \\
& \forall r \in O, \\
& \sum_{j \in \Pi} \lambda_{j}+\lambda_{q}=1, & \\
& \beta_{r j}^{u} \geq y_{r j}^{u}, & \\
& \lambda_{j} \geq 0, j \in \Pi \cup\{q\}, & \forall j \in I, \forall j .
\end{array}
$$

In model (3.5), $\left(\lambda_{j} ; j \in \Pi \cup\{q\}, \beta_{r j}^{u} ; \forall r \in O, \forall j \in J\right)$ is the variables vector. In the above model, the expected efficiency lower bound for $\mathrm{DMU}_{q}$ is denoted by $\bar{\varphi}_{q}^{l}$. The objectives of the MOP (3.5) guarantees that the outputs inherited by the merged DMU from the participating units in the merger are maximized, in order to realize the desired efficiency target $\bar{\varphi}_{q}^{l}$. In other words, the main motive for M\&A is to reach the maximum benefit by maximizing outputs in the current section. According to a similar reasoning for converting model (3.1) to model (3.2), model (3.5) could be converted to model (3.6), as well. The proof of the following theorem is omitted because it is similar to the proof of Theorem 3.1 .

Theorem 3.5. If DMU ${ }_{q}$ is within the current PPS, then model (3.5) can be converted to the following MOLP problem:

$$
\begin{array}{lll}
\max & \left(\beta_{r j}^{u} ; \forall r \in O, \forall j \in \Lambda\right) & \\
\text { s.t. } & \sum_{j \in \Pi} \lambda_{j} x_{i j}^{l} \leq x_{i q}^{l}, & \forall i \in I, \\
& \sum_{j \in \Pi} \lambda_{j} y_{r j}^{u} \geq \bar{\varphi}_{q}^{l} \sum_{j \in \Lambda} \beta_{r j}^{u}, & \forall r \in O, \\
& \sum_{j \in \Pi} \lambda_{j}=1, & \\
& \beta_{r j}^{u} \geq y_{r j}^{u}, & \forall i \in I, \forall j \in \Lambda, \\
& \lambda_{j} \geq 0, & \forall j \in \Pi .
\end{array}
$$

Theorem 3.6 shows how the above model could be utilized to identification of the upper limit of the $\mathrm{DMU}_{q}$ output levels.

Theorem 3.6. If the following assumptions hold:

(i) The merged unit (DMUq) is within the current PPS;

(ii) $\Delta^{u}=\left(\lambda^{*}, \beta_{r j}^{u *}: \forall r \in O, \forall j \in \Lambda\right)$ is a weak Pareto solution to problem (3.6);

(iii) $y_{r q}^{u}=\sum_{j \in \Lambda} \beta_{r j}^{u *}$ for each $r \in O$.

Then, the obtained efficiency lower bound of $D M U_{q}$ is equal to $\bar{\varphi}_{q}^{l}$.

Proof. See Appendix C. 
To estimate of the lower limit of the input levels of $\mathrm{DMU}_{q}$ to achieve the desired given efficiency target $\bar{\varphi}_{q}^{u}$, the following MOP model is proposed:

$$
\begin{aligned}
& \max \quad\left(\beta_{r j}^{l} ; \forall r \in O, \forall j \in \Lambda\right) \\
& \text { s.t. } \quad \sum_{j \in \Pi} \lambda_{j} x_{i j}^{l}+x_{i q}^{l} \lambda_{q} \leq x_{i q}^{u}, \quad \forall i \in I, \\
& \sum_{j \in \Pi} \lambda_{j} y_{r j}^{u}+\left(\sum_{j \in \Lambda} \bar{\beta}_{r j}^{u}\right) \lambda_{q} \geq \bar{\varphi}_{q}^{u} \sum_{j \in \Lambda} \beta_{r j}^{l}, \quad \forall r \in O, \\
& \sum_{j \in \Pi} \lambda_{j}+\lambda_{q}=1 \\
& y_{r j}^{l} \leq \beta_{r j}^{l} \leq \bar{\beta}_{r j}^{u}, \quad \forall r \in O, \forall j \in \Lambda, \\
& \lambda_{j} \geq 0, \\
& \forall j \in \Pi \text {. }
\end{aligned}
$$

In model (3.7), $\left(\lambda_{j} ; j \in \Pi \cup\{q\}, \beta_{r j}^{l} ; \forall r \in O, \forall j \in J\right)$ is the variables vector. In this model, the expected efficiency lower bound for $\mathrm{DMU}_{q}$ is denoted by $\bar{\varphi}_{q}^{u} \cdot\left(\bar{\beta}_{r j}^{u} ; \forall j \in \Lambda, \forall r \in O\right)$ is a Pareto solution of model (3.6). The following theorem shows how the above MOP can be linearized.

Theorem 3.7. If $D M U_{q}$ is within the current PPS, then model (3.5) can be converted to the following MOLP problem:

$$
\begin{array}{lll}
\max & \left(\beta_{r j}^{l} ; \forall r \in O, \forall j \in \Lambda\right) & \\
\text { s.t. } & \sum_{j \in \Pi} \lambda_{j} x_{i j}^{l} \leq x_{i q}^{u}, & \forall i \in I, \\
& \sum_{j \in \Pi} \lambda_{j} y_{r j}^{u} \geq \bar{\varphi}_{q}^{u} \sum_{j \in \Lambda} \beta_{r j}^{l}, & \forall r \in O, \\
& \sum_{j \in \Pi} \lambda_{j}=1, & \\
& y_{r j}^{l} \leq \beta_{r j}^{l} \leq \bar{\beta}_{r j}^{u}, & \forall r \in O, \forall j \in \Lambda, \\
& \lambda_{j} \geq 0, & \forall j \in \Pi .
\end{array}
$$

Proof. The proof is similar to the proof of Theorem 3.1. Note that the best situation of $\mathrm{DMU}_{q}$ $\left(x_{i q}^{l}, y_{r q}^{u}=\sum_{j \in \Lambda} \bar{\beta}_{r j}^{u}\right)$ could be stated by a convex combination of some the best situation of units that have not participated in merger.

The following theorem shows how the MOLP (3.8) could be used to estimate of the lower limit of the $\mathrm{DMU}_{q}$ output levels.

Theorem 3.8. If the following assumptions hold:

(i) The merged unit (DMUq) is within the current PPS;

(ii) $\Delta^{l}=\left(\lambda^{*}, \beta_{r j}^{l *}: \forall r \in O, \forall j \in \Lambda\right)$ is a Pareto solution to model (3.8) in which $\bar{\beta}_{r j}^{u} \neq \beta_{r j}^{l *}$;

(iii) $y_{r q}^{l}=\sum_{j \in \Lambda} \beta_{r j}^{l *}$ for each $r \in O$.

Then, the obtained efficiency upper bound of $D M U_{q}$ is equal to $\bar{\varphi}_{q}^{u}$.

Proof. See Appendix D. 
Remark 3.9. In our study, the problem of merging units is developed provided that the merged unit is within the current PPS. It is obvious that the $\mathrm{DMU}_{q}$ will be inside of the current PPS, if and only if the virtual DMU $\left(\left[\sum_{j \in \Lambda} x_{i j}^{l}, \sum_{j \in \Lambda} x_{i j}^{u}\right],\left[\sum_{j \in \Lambda} y_{r j}^{l}, \sum_{j \in \Lambda} y_{r j}^{u}\right]\right)$ for all $r \in O$ and $i \in I$; is within the PPS. This arises from the goals of the MOP input-(resp. output-) oriented models (3.1) and (3.3) (resp. (3.5) and (3.7)) as well as the goals of the relaxed input-(resp. output-) oriented models (3.2) and (3.4) (resp. (3.6) and (3.8)). This assumption guarantees that the merged unit is inside the PPS when it is inefficient or on the frontier once it is efficient. It is worth noting that the models (3.1) to (3.4) try to keep the minimum level of the inputs of units $j \in \Lambda$, while models (3.5) to (3.8) try to keep the maximum level of the outputs of units $j \in \Lambda$. Therefore, without loss of generality, we can consider the models (3.2), (3.4), (3.6), and (3.8) instead of models (3.1), (3.3), (3.5), and (3.7), respectively.

\section{Minimum And maXimum aCHIEVABle EFFiCIEnCy targets}

This section discusses about best and lowest values of lower and upper bounds of achievable efficiency targets of the merged DMU. In fact, this section is devoted to answering Question 2. A method is proposed for finding the pessimistic and optimistic levels of the achievable efficiency targets that can be attained through the merged DMU. Such a method can be informative because it provides foresight about the post-merging market positioning and the minimum and maximum efficiency level it can achieve. It is worth noting that knowing minimum and maximum achievable efficiency targets has an important role for assessment of the decision maker about engaging in the merging process.

In Sections 4.1 and 4.2 are investigated identification of the minimum and maximum achievable efficiency targets that can be attained through the merged DMU.

\subsection{Minimum and maximum achievable targets of the input-oriented efficiency}

According to discussion of the Section 3.1, $\mathrm{DMU}_{q}$ keeps the amount of outputs of set of the merging DMUs and looks for the minimum amount of the inherited sources of these DMUs in order to reach the pre-defined target level, $\bar{\theta}_{q}=\left[\bar{\theta}_{q}^{l}, \bar{\theta}_{q}^{u}\right]$. Knowing lowest and highest achievable efficiency targets has a critical importance for the merging' decision maker. Because, it is the prediction about the position of the merged unit. To attain these goals, suppose that the maximum value of lower and upper bounds of achievable efficiency targets of the merged DMU are denoted by $\bar{\theta}_{q}^{l-\max }$ and $\bar{\theta}_{q}^{u-\max }$, respectively. In addition, assume that the minimum value of lower and upper bounds of achievable efficiency targets of the merged DMU are denoted by $\bar{\theta}_{q}^{l-m i n}$ and $\bar{\theta}_{q}^{u-\min }$, respectively. In fact, it is assumed that the pessimistic and optimistic levels of the attainable efficiency through this merge is equal to $\left[\bar{\theta}_{q}^{l-\min }, \bar{\theta}_{q}^{l-\max }\right]$ and $\left[\bar{\theta}_{q}^{u-\min }, \bar{\theta}_{q}^{u-\max }\right]$, respectively. Clearly, if these pessimistic and optimistic levels are satisfactory, then the decision maker is encouraged to merge. This section is dedicated to identifying the pessimistic and optimistic levels that can be attained through the merged DMU.

The optimistic level of the attainable efficiency score through the merged DMU, could be determined through the following theorem.

Theorem 4.1. Suppose that the model (3.2) is feasible for the efficiency target $\bar{\theta}_{q}^{u}$. Then

(i) Model (3.2) remains feasible for each efficiency upper bound target $\hat{\theta}_{q}^{u}$, where $\bar{\theta}_{q}^{u} \leq \hat{\theta}_{q}^{u} \leq 1$.

(ii) If

$$
\begin{array}{ll}
\theta_{q}^{u *}= & \min \theta \\
\text { s.t. } & \sum_{j \in \Pi} \lambda_{j} x_{i j}^{l} \leq \sum_{j \in \Lambda} \alpha_{i j}^{l}, \quad \forall i \in I, \\
& \sum_{j \in \Pi} \lambda_{j} y_{r j}^{u} \geq y_{r q}^{u}, \quad \forall r \in O,
\end{array}
$$




$$
\begin{array}{ll}
\sum_{j \in \Pi} \lambda_{j}=1, & \\
0 \leq \alpha_{i j}^{l} \leq x_{i j}^{l}, & \forall i \in I, \forall j \in \Lambda, \\
\sum_{j \in \Lambda} \alpha_{i j}^{l} \leq \theta \sum_{j \in \Lambda} x_{i j}^{l}, & \forall i \in I, \\
\lambda_{j} \geq 0, & \forall j \in \Pi,
\end{array}
$$

then $\theta_{q}^{u *} \leq \bar{\theta}_{q}^{u}$ (the minimum achievable upper bound for the efficiency target of the merged DMU is $\theta_{q}^{u *}$, i.e. $\left.\bar{\theta}_{q}^{u-\min }=\theta_{q}^{u *}\right)$.

Remark 4.2. The last set of the constraints in the model (4.1) guarantees that the sum of the lower bounds of the allocated inherited inputs to the merged unit is at most equal to the product of the minimum realized upper bound of efficiency score by the aggregated inputs of the participating units in the merger.

Proof. It is obvious that any feasible solution of the model (3.2) corresponding to the efficiency target $\bar{\theta}_{q}^{u}$, is a feasible solution of the model corresponding to the efficiency target $\hat{\theta}_{q}^{u}$, where $\bar{\theta}_{q}^{u} \leq \hat{\theta}_{q}^{u} \leq 1$. This completes the proof of part (i).

By contradiction assume that the model (3.2) is feasible for merging with the efficiency upper bound target $\bar{\theta}_{q}^{u}$ such that $\bar{\theta}_{q}^{u}<\theta_{q}^{u *}$. Let $\Delta^{l}=\left(\lambda^{*}, \alpha_{i j}^{l *}: \forall i \in I, \forall j \in \Lambda\right)$ be a Pareto solution to model (3.2). Feasibility of $\Delta^{l}$ to model (3.2), implies:

$$
\alpha_{i j}^{l *} \leq x_{i j}^{l}, \quad \forall i \in I, \quad \forall j \in \Lambda .
$$

By (4.2) and $0<\bar{\theta}_{q}^{u} \leq 1$, we get

$$
\begin{gathered}
\bar{\theta}_{q}^{u} \alpha_{i j}^{l *} \leq \alpha_{i j}^{l *} \leq x_{i j}^{l}, \quad \forall i \in I, \quad \forall j \in \Lambda, \\
\sum_{j \in \Lambda} \bar{\theta}_{q}^{u} \alpha_{i j}^{l *} \leq \sum_{j \in \Lambda} \bar{\theta}_{q}^{u} x_{i j}^{l}=\bar{\theta}_{q}^{u} \sum_{j \in \Lambda} x_{i j}^{l}, \quad \forall i \in I .
\end{gathered}
$$

Now, for all $i \in I$ and $j \in \Lambda$ define $\bar{\alpha}_{i j}^{l}=\bar{\theta}_{q}^{u} \alpha_{i j}^{l *}$. By (4.3), (4.4), and feasibility of $\Delta^{l}$ to model (3.2), it is obvious that $\Psi=\left(\lambda^{*}, \bar{\theta}_{q}^{u}, \bar{\alpha}_{i j}^{l}: \forall i \in I, \forall j \in \Lambda\right)$ is a feasible solution to model (4.1). Therefore, the optimal value of model (4.1) is less or equal $\bar{\theta}_{q}^{u}$. This contradicts the assumption that $\theta_{q}^{u *}$ is the optimal value of model (4.1) and so the proof of part (ii) is completed.

The pessimistic level of the attainable efficiency score through the merged DMU, could be determined through the Theorems 4.3 and 4.4. In other words, the minimum and maximum achievable lower bound of the efficiency target through the generated new unit, i.e. $\bar{\theta}_{q}^{l-\min }$ and $\bar{\theta}_{q}^{l-\max }$, could be attained through the Theorems 4.3 and 4.4, respectively. It is worth noting that the decision of merging can be made if this pessimistic level is judged satisfactory.

Theorem 4.3. Suppose that model (3.4) is feasible for the efficiency target $\bar{\theta}_{q}^{l}$. If

$$
\begin{aligned}
\theta_{q}^{l *}= & \min \theta \\
\text { s.t. } & \sum_{j \in \Pi} \lambda_{j} x_{i j}^{l} \leq \sum_{j \in \Lambda} \alpha_{i j}^{u}, \quad \forall i \in I, \\
& \sum_{j \in \Pi} \lambda_{j} y_{r j}^{u} \geq y_{r q}^{l}, \quad \forall r \in O, \\
& \sum_{j \in \Pi} \lambda_{j}=1,
\end{aligned}
$$




$$
\begin{array}{ll}
\sum_{j \in \Lambda} \alpha_{i j}^{u} \leq \theta \sum_{j \in \Lambda} x_{i j}^{u}, & \forall i \in I, \\
\lambda_{j} \geq 0, & \forall j \in \Pi,
\end{array}
$$

then

(i) $\theta_{q}^{l *} \leq \theta_{q}^{u *}$, where $\theta_{q}^{u *}$ is the optimal solution of model (4.1).

(ii) $\theta_{q}^{l *} \leq \bar{\theta}_{q}^{l}$ (the minimum achievable lower bound efficiency target of the merged DMU is $\theta_{q}^{l *}$, i.e. $\bar{\theta}_{q}^{l-\min }=\theta_{q}^{l *}$ ).

Proof. Let $\Omega=\left(\lambda^{*}, \theta^{*}, \alpha_{i j}^{l *} ; \forall i \in I, \forall j \in \Pi\right)$ be a optimal solution to model (4.1). Since $x_{i j}^{l} \leq x_{i j}^{u}$ and $y_{r j}^{l} \leq y_{r j}^{u}$ for all $i, r, j$; it is obvious that $\Omega$ is a feasible solution to model (4.5) ( $\operatorname{setting} \lambda=\lambda^{*}, \theta=\theta^{*}, \alpha_{i j}^{u}=\alpha_{i j}^{l *}$ ). Therefore, $\theta_{q}^{l *} \leq \theta_{q}^{u *}$ and so the proof of part (i) is completed. In the similar method to the proof of part (ii) of Theorem 4.1, we can show that the $\theta_{q}^{l *} \leq \bar{\theta}_{q}^{l}$.

Theorem 4.4. Let a merging with $\bar{\theta}_{q}^{l}$ and $\bar{\theta}_{q}^{u}$ be as the efficiency lower and upper bounds targets for the merged DMU. Let $\left(\bar{\alpha}_{i j}^{l} ; \forall i \in I, \forall j \in \Lambda\right)$ be a Pareto solution of MOLP (3.2). Moreover, suppose that model (3.4) is feasible for the efficiency target $\bar{\theta}_{q}^{l}$. If $\theta_{q}^{l *}$ is the optimal value of the following model:

$$
\begin{aligned}
& \min \theta_{q}^{l} \\
& \text { s.t. } \quad \sum_{j \in \Pi} \lambda_{j} x_{i j}^{l} \leq \theta_{q}^{l} x_{i q}^{l}=\theta_{q}^{l} \sum_{j \in \Lambda} \bar{\alpha}_{i j}^{l}, \quad i=1,2, \ldots, m, \\
& \sum_{j \in \Pi} \lambda_{j} y_{r j}^{u} \geq y_{r q}^{l}, \quad r=1,2, \ldots, s, \\
& \sum_{j \in \Pi} \lambda_{j}=1, \\
& \lambda_{j} \geq 0, \quad \forall j \in \Pi,
\end{aligned}
$$

then model (3.4) remains feasible for each efficiency target $\hat{\theta}_{q}^{l}$, where $\bar{\theta}_{q}^{l} \leq \hat{\theta}_{q}^{l} \leq \theta_{q}^{l *}$ (the maximum achievable lower bound efficiency target of the merged $D M U$ is $\theta_{q}^{l *}$, i.e. $\left.\bar{\theta}_{q}^{l-m a x}=\theta_{q}^{l *}\right)$.

Proof. The proof is straightforward.

\subsection{Minimum and maximum achievable targets of the output-oriented efficiency}

This section is devoted to answering Question 2 when the efficiency score is estimated using the outputoriented models. To attain this goal, a similar process to the previous section is used. According to discussion of the Section 3.2, $\mathrm{DMU}_{q}$ keeps the amount of inputs of set of the merging units and looks for the maximum amount of the inherited outputs of these units to reach the pre-defined target level, $\bar{\varphi}_{q}=\left[\bar{\varphi}_{q}^{l}, \bar{\varphi}_{q}^{u}\right]$. As previously mentioned, finding the pessimistic and optimistic levels of the achievable efficiency targets that can be attained through the merged unit has a critical importance for the merging' decision maker. To attain these goals, it is assumed that the optimistic and pessimistic levels of the attainable efficiency through this merge is equal to $\left[\bar{\varphi}_{q}^{l-\min }, \bar{\varphi}_{q}^{l-\max }\right]$ and $\left[\bar{\varphi}_{q}^{u-\min }, \bar{\varphi}_{q}^{u-\max }\right]$, respectively. In fact, it is assumed that the minimum value of lower and upper bounds of achievable efficiency targets of the merged DMU are denoted by $\bar{\varphi}_{q}^{l-\min }$ and $\bar{\varphi}_{q}^{u-\min }$, respectively. Moreover, it is assumed that the maximum value of lower and upper bounds of achievable efficiency targets of the merged DMU are denoted by $\bar{\varphi}_{q}^{l-\max }$ and $\bar{\varphi}_{q}^{u-\max }$, respectively. It is worth noting that the decision of merging can be made if these pessimistic and optimistic levels are judged satisfactory.

The optimistic level of the achievable efficiency score through the merged unit, could be attained through the following theorem. 
Theorem 4.5. Suppose that the model (3.6) is feasible for the efficiency target $\bar{\varphi}_{q}^{l}$. Then

(i) Model (3.6) remains feasible for each efficiency upper bound target $\hat{\varphi}_{q}^{l}$, where $1 \leq \hat{\varphi}_{q}^{l} \leq \bar{\varphi}_{q}^{l}$.

(ii) If

$$
\begin{aligned}
& \varphi_{q}^{l *}=\max \varphi \\
& \text { s.t. } \quad \sum_{j \in \Pi} \lambda_{j} x_{i j}^{l} \leq x_{i q}^{l}, \quad \forall i \in I, \\
& \sum_{j \in \Pi} \lambda_{j} y_{r j}^{u} \geq \sum_{j \in \Lambda} \beta_{r j}^{u}, \quad \forall r \in O, \\
& \sum_{j \in \Pi} \lambda_{j}=1, \\
& \beta_{r j}^{u} \geq y_{r j}^{u}, \quad \forall r \in O, \forall j \in \Lambda, \\
& \sum_{j \in \Lambda} \beta_{r j}^{u} \geq \varphi \sum_{j \in \Lambda} y_{r j}^{u}, \quad \forall r \in O, \\
& \lambda_{j} \geq 0, \quad \forall j \in \Pi \text {. }
\end{aligned}
$$

then $\varphi_{q}^{l *} \geq \bar{\varphi}_{q}^{l}$ (the maximum achievable lower bound for the efficiency target of the merged DMU is $\varphi_{q}^{l *}$, i.e. $\left.\bar{\varphi}_{q}^{l-\max }=\varphi_{q}^{l *}\right)$.

Remark 4.6. The last set of the constraints in the model (4.7) guarantees that the sum of the upper bounds of the productive inherited outputs by the merged unit is at least equal to the product of the maximum realized lower bound of efficiency score by the aggregated outputs of the participating units in the merger.

Proof. It is obvious that any feasible solution of the model (3.6) corresponding to the efficiency target $\bar{\varphi}_{q}^{l}$, is a feasible solution of the model corresponding to the efficiency target $\varphi_{q}^{l *}$, where $1 \leq \hat{\varphi}_{q}^{l} \leq \bar{\varphi}_{q}^{l}$. This completes the proof of part (i).

By contradiction assume that the model (3.6) is feasible for merging with the efficiency upper bound target $\bar{\varphi}_{q}^{l}$ such that $\varphi_{q}^{l *}<\bar{\varphi}_{q}^{l}$. Let $\Delta^{u}=\left(\lambda^{*}, \beta_{r j}^{u *}: \forall r \in O, \forall j \in \Lambda\right)$ be a Pareto solution to model (3.6). Feasibility of $\Delta^{u}$ to model (3.6), implies:

$$
\beta_{r j}^{u *} \geq y_{r j}^{u}, \quad \forall r \in O, \forall j \in \Lambda .
$$

By (4.8) and $\bar{\varphi}_{q}^{l} \geq 1$, we get

$$
\begin{aligned}
\bar{\varphi}_{q}^{l} \beta_{r j}^{u *} & \geq \beta_{r j}^{u *} \geq y_{r j}^{u}, \quad \forall r \in O, \forall j \in \Lambda, \\
\sum_{j \in \Lambda} \bar{\varphi}_{q}^{l} \beta_{r j}^{u *} & \geq \sum_{j \in \Lambda} \bar{\varphi}_{q}^{l} y_{r j}^{u}=\bar{\varphi}_{q}^{l} \sum_{j \in \Lambda} y_{r j}^{u}, \quad \forall r \in O .
\end{aligned}
$$

Now, for all $r \in O$ and $j \in \Lambda$ define $\bar{\beta}_{r j}^{u}=\bar{\varphi}_{q}^{l} \beta_{r j}^{u *}$. By (4.9), (4.10), and feasibility of $\Delta^{u}$ to model (3.6), it is obvious that $\Psi=\left(\lambda^{*}, \bar{\varphi}_{q}^{l}, \bar{\beta}_{r j}^{u}: \forall r \in O, \forall j \in \Lambda\right)$ is a feasible solution to model (4.7). Therefore, the optimal value of model (4.7) is less or equal $\bar{\varphi}_{q}^{l}$. This contradicts the assumption that $\varphi_{q}^{l *}$ is the optimal value of model (4.7) and so the proof of part (ii) is completed.

The pessimistic level of the achievable efficiency score through the merged DMU, could be attained through the Theorems 4.7 and 4.8. In other words, the maximum and minimum values of upper bound of achievable efficiency targets of the merged DMU, i.e. $\bar{\varphi}_{q}^{u-\max }$ and $\bar{\varphi}_{q}^{u-\min }$, could be attained through the the Theorems 4.7 and 4.8 , respectively. 
Theorem 4.7. Suppose that model (3.8) is feasible for the efficiency target $\bar{\varphi}_{q}^{u}$. If

$$
\begin{aligned}
& \varphi_{q}^{u *}=\max \varphi \\
& \text { s.t. } \quad \sum_{j \in \Pi} \lambda_{j} x_{i j}^{l} \leq x_{i q}^{u}, \quad \forall i \in I, \\
& \sum_{j \in \Pi} \lambda_{j} y_{r j}^{u} \geq \sum_{j \in \Lambda} \beta_{r j}^{l}, \quad \forall r \in O, \\
& \sum_{j \in \Pi} \lambda_{j}=1 \text {, } \\
& \beta_{r j}^{l} \geq y_{r j}^{l}, \quad \forall r \in O, \forall j \in \Lambda, \\
& \sum_{j \in \Lambda} \beta_{r j}^{l} \geq \varphi \sum_{j \in \Lambda} y_{r j}^{l}, \quad \forall r \in O, \\
& \lambda_{j} \geq 0, \quad \forall j \in \Pi \text {. }
\end{aligned}
$$

then

(i) $\varphi_{q}^{u *} \geq \varphi_{q}^{l *}$, where $\varphi_{q}^{l *}$ is the optimal solution of model (4.7).

(ii) $\varphi_{q}^{u *} \geq \bar{\varphi}_{q}^{u}$ (the maximum achievable upper bound for the efficiency target of the merged DMU is $\varphi_{q}^{u *}$, i.e. $\left.\bar{\varphi}_{q}^{u-\max }=\varphi_{q}^{u *}\right)$.

Proof. Let $\Omega=\left(\lambda^{*}, \varphi^{*}, \beta_{r j}^{u *} ; \forall r \in O, \forall j \in \Pi\right)$ be a optimal solution to model (4.7). Since $x_{i j}^{l} \leq x_{i j}^{u}$ and $y_{r j}^{l} \leq y_{r j}^{u}$ for all $\mathrm{i}, \mathrm{r}, \mathrm{j}$; it is obvious that $\Omega$ is a feasible solution to model (4.11) (setting $\lambda=\lambda^{*}, \varphi=\varphi^{*}, \beta_{r j}^{l}=\beta_{r j}^{u *}$ ). Therefore, $\varphi_{q}^{u *} \geq \varphi_{q}^{l *}$ and so the proof of part (i) is completed. In the similar method to the proof of part (ii) of Theorem 4.5, we can show that the $\varphi_{q}^{u *} \geq \bar{\varphi}_{q}^{u}$.

Theorem 4.8. Let a merging with $\bar{\varphi}_{q}^{u}$ and $\bar{\varphi}_{q}^{l}$ be as the efficiency lower and upper bounds targets for the merged DMU. Let $\left(\bar{\beta}_{r j}^{u} ; \forall r \in O, \forall j \in \Lambda\right)$ be a Pareto solution of MOLP (3.6). Moreover, suppose that model (3.8) is feasible for the efficiency target $\bar{\varphi}_{q}^{u}$. If $\varphi_{q}^{u *}$ is the optimal value of the following model:

$$
\begin{array}{lll}
\max & \varphi_{q}^{u} & \\
\text { s.t. } & \sum_{j \in \Pi} \lambda_{j} x_{i j}^{l} \leq x_{i q}^{u}, & i=1,2, \ldots, m, \\
& \sum_{j \in \Pi} \lambda_{j} y_{r j}^{u} \geq \varphi_{q}^{u} y_{r q}^{l}=\varphi_{q}^{u} \sum_{j \in \Lambda} \bar{\beta}_{r j}^{u}, & \\
& \sum_{j \in \Pi} \lambda_{j}=1, & \\
& \lambda_{j} \geq 0, & \forall j \in \Pi, 2, \ldots, s,
\end{array}
$$

then model (3.8) remains feasible for each efficiency target $\hat{\varphi}_{q}^{u}$, where $\varphi_{q}^{u *} \leq \hat{\varphi}_{q}^{u} \leq \bar{\varphi}_{q}^{u}$ (the minimum achievable upper bound for the efficiency target of the merged DMU is $\varphi_{q}^{u *}$, i.e. $\left.\bar{\varphi}_{q}^{u-\min }=\varphi_{q}^{u *}\right)$.

Proof. The proof is straightforward.

Remark 4.9. An important issue for decision maker involved in a merging program would be to know the minimum and maximum efficiency score that can be attained by the merged unit. Section 4 addresses this issue. More precisely, an insightful method is provided to obtain the minimum and maximum achievable lower and 
TABLE 1 . The efficiency interval of 20 bank branches.

\begin{tabular}{llll}
\hline \hline Branches & Efficiency interval & Branches & Efficiency interval \\
\hline B01 & {$[0.88,1.00]$} & B11 & {$[0.95,1.00]$} \\
B02 & {$[0.79,0.90]$} & B12 & {$[0.62,0.67]$} \\
B03 & {$[0.73,1.00]$} & B13 & {$[0.68,0.85]$} \\
B04 & {$[0.90,1.00]$} & B14 & {$[0.64,0.71]$} \\
B05 & {$[0.96,1.00]$} & B15 & {$[0.47,1.00]$} \\
B06 & {$[0.90,1.00]$} & B16 & {$[0.57,0.66]$} \\
B07 & {$[0.77,1.00]$} & B17 & {$[0.99,1.00]$} \\
B08 & {$[0.90,1.00]$} & B18 & {$[0.98,1.00]$} \\
B09 & {$[0.87,1.00]$} & B19 & {$[0.86,1.00]$} \\
B10 & {$[0.98,1.00]$} & B20 & {$[0.50,0.66]$} \\
\hline
\end{tabular}

TABLE 2. The inherited outputs of the branch $B_{q}$ from the merging branches B12 and B14.

\begin{tabular}{llllll}
\hline \hline Branches & $\begin{array}{l}\text { The total sum of } \\
\text { four main deposits }\end{array}$ & Other deposits & Loans granted & Received interest & Fee \\
\hline B12 & {$[453170,481943]$} & {$[27196,29553]$} & {$[245726,275717]$} & {$[35757.83,42790.14]$} & {$[375.07,559.85]$} \\
B14 & {$[309670,342598]$} & {$[20168,26172]$} & {$[124188,126930]$} & {$[8143.79,11948.04]$} & {$[936.62,1468.45]$} \\
Bq & {$[762840,824541]$} & {$[47364,55725]$} & {$[369914,402647]$} & {$[43901.62,54738.18]$} & {$[1311.69,2028.3]$} \\
\hline
\end{tabular}

upper bounds of the efficiency score of the merged unit. In other words, input-(resp. output-) oriented models (4.1), (4.5), and (4.6) (resp. (4.7), (4.11), and (4.12)) are provided to obtain the lowest and highest achievable lower and upper bounds of the efficiency interval of the merged unit can realize. The results of these models have a critical importance for the merging' manager. The results are very informative because these provide foresight about the position of the merged unit. These minimum and maximum values could be considered as thresholds for the merger approval if these values are satisfactory.

\section{A NUMERICAL illustration}

In this section, the performance of the proposed approach is demonstrated through an example in banking sector. Nevertheless, this approach could be used in other sectors with the merging possibility. We consider 20 commercial bank branches in Iran. As we know, there are two main approaches (the production and the intermediation approach) to select of the input and output factors. In this study, the input and output factors are selected based on the intermediation approach. Each branch produces five outputs using three inputs. The total sum of four main deposits $\left(y_{1}\right)$, other deposits $\left(y_{2}\right)$, loans granted $\left(y_{3}\right)$, received interest $\left(y_{4}\right)$, and fee $\left(y_{5}\right)$ are considered as outputs while the payable interest $\left(x_{1}\right)$, personnel $\left(x_{2}\right)$, and non-performing loans $\left(x_{3}\right)$ are considered as the input factors. The dataset is reproduced from Jahanshahloo et al. [31] and presented in Appendix E.

To estimate the lower and upper bounds of the efficiency of 20 bank branches, models (2.2) and (2.3) are applied. The results are reported in Table 1.

According to Definition of 2.2, B12 and B14 branches are inefficient (see Tab. 1). Assume that these branches consolidate their activities by producing the new merged branch q $\left(B_{q}\right)$ such that $B_{q}$ keeps the amount of outputs of branches of B12 and B14 and looks for the minimum amount of the inherited inputs of these branches to reach the pre-defined target level. According to this discussion, the output levels of the new branch $B_{q}$ are obtained and it is presented in Table 2. 
TABLE 3. The inherited inputs of the branch $B_{q}$ of the merging branches B12 and B14.

\begin{tabular}{llll}
\hline \hline Branches & Payable interest & Personnel & Non-performing loans \\
\hline B12 & {$[7303.27,14178.11]$} & {$[22.87,23.19]$} & {$[16148,21353]$} \\
B14 & {$[4540.75,9312.24]$} & {$[22.83,23.96]$} & {$[17918,17964]$} \\
Bq & {$[3551.75,3577.72]$} & {$[25.73,29.09]$} & {$[6669.16,6669.16]$} \\
\hline
\end{tabular}

According to model (4.1), the lowest attainable efficiency for the upper bound by the merged branch $B_{q}$ is $\theta_{q}^{u}=0.43$. According to Theorem 4.1, if the condition $\bar{\theta}_{q}^{u} \geq 0.43$ is violated, then the corresponding model (3.2) becomes infeasible. Based on model (4.5), the minimum achievable efficiency for the lower bound by the merged branch $B_{q}$ is obtained as $\theta_{q}^{l}=0.37$. Therefore, the least attainable efficiency level through this merge is equal to $[0.37,0.43]$. If this pessimistic level is satisfactory, the decision maker is encouraged to perform the merging.

According to Theorem 4.1, the maximum attainable efficiency for the upper bound by the merged branch $B_{q}$ is equal to $\theta_{q}^{u}=1$. According to Theorem 4.4, if the efficiency upper bound target of the merged branch $B_{q}$ is equal to $\theta_{q}^{u}=1$, then the maximum achievable efficiency for the lower bound by the merged branch $B_{q}$ could be obtained as $\theta_{q}^{l}=0.96$. Therefore, the most attainable efficiency level through this merge is equal to $[0.96,1.00]$. In fact, this is an optimistic level for this merger.

The branches B12 and B14 are merged with two different expected performance levels. First, suppose that the efficiency target of the merged branch $B_{q}$ is equal to $\bar{\theta}_{q}=\left[\bar{\theta}_{q}^{l}, \bar{\theta}_{q}^{u}\right]=[0.7,0.80]$. To identify the minimum inherited input levels of the $B_{q}$ from merging units (B12 and B14), the proposed model (3.2) that corresponds to this merger is considered.

The weight-sum method [14] is employed to generate different Pareto solutions for this model. It is worth noting that if there is no priority in reducing different inputs, then we can consider the same weights for all the inputs. However, if the decision maker seeks various aims from merging these branches (such as saving more inputs from a special DMU), then the weights should be considered differently. Here, due to simplicity, we consider the same weights for all the objective functions and a Pareto solution is generated as follows:

$$
\begin{aligned}
& \alpha_{1,12}^{l *}=3551.75, \alpha_{2,12}^{l *}=22.87, \alpha_{3,12}^{l *}=6669.16 \\
& \alpha_{1,14}^{l *}=0.00, \quad \alpha_{2,14}^{l *}=2.86, \quad \alpha_{3,14}^{l *}=0.00 \\
& \lambda_{1}^{*}=0.02, \quad \lambda_{4}^{*}=0.15, \quad \lambda_{10}^{*}=0.04, \quad \lambda_{11}^{*}=0.79, \quad \lambda_{j}^{*}=0 \quad \forall j \neq 1,4,10,11 .
\end{aligned}
$$

Accordingly, the lower bounds of the inherited input levels of the $\mathrm{Bq}$ from merging branches (B12 and B14) to reach the upper pre-defined target level $\left(\bar{\theta}_{q}^{u}=0.80\right)$ are extracted (see Tab. 3). The obtained Pareto solution (5.1) is utilized in Theorem 4.4 to obtain the maximum achievable efficiency lower bound by the merged branch $B_{q}$ as $\theta_{q}^{l}=0.77$. Therefore, the intended efficiency lower bound $\bar{\theta}_{q}^{l}=0.70$ of the merged branch $B_{q}$ is achievable. To estimate the minimum upper bound of the inherited input levels from merging branches (B12 and B14), the proposed model (3.4) that corresponds with this merger is considered. Using the weight-sum method, we obtained a Pareto solution for this MOLP as:

$$
\begin{array}{lll}
\alpha_{1,12}^{u *}=3577.72, & \alpha_{2,12}^{u *}=23.19, & \alpha_{3,12}^{u *}=6669.16 \\
\alpha_{u, 14}^{l *}=0.00, & \alpha_{2,14}^{u *}=5.90, & \alpha_{3,14}^{u *}=0.00 \\
\lambda_{4}^{*}=0.21, & \lambda_{10}^{*}=0.02, \quad \lambda_{11}^{*}=0.77, \quad \lambda_{j}^{*}=0 & \forall j \neq 4,10,11 .
\end{array}
$$

Accordingly, the upper bounds of the inherited input levels the Bq from merging branches (B12 and B14) to achieve the lower pre-defined target level $\left(\theta_{q}^{l}=0.70\right)$ are extracted (see Tab. 3).

As another intended performance level, consider that the merger of two merging branches B12 and B14 should achieve an optimistic target level, that is equal to [0.96, 1.00]. By using models $(3.2)$ and (3.4) that correspond 
TABLE 4 . The inherited inputs of the branch $B_{q}$ of the merging branches B12 and B14.

\begin{tabular}{llll}
\hline \hline Branches & Payable interest & Personnel & Non-performing loans \\
\hline B12 & {$[7303.27,14178.11]$} & {$[22.87,23.19]$} & {$[16148,21353]$} \\
B14 & {$[4540.75,9312.24]$} & {$[22.83,23.96]$} & {$[17918,17964]$} \\
Bq & {$[2841.40,2841.40]$} & {$[20.58,20.60]$} & {$[5335.33,5335.33]$} \\
\hline
\end{tabular}

with this merger, the following two Pareto solutions are generated for these models, respectively;

$$
\begin{aligned}
& \alpha_{1,12}^{l *}=2841.40, \alpha_{2,12}^{l *}=20.58, \alpha_{3,12}^{l *}=5335.33 \\
& \alpha_{1,14}^{l *}=0.00, \quad \alpha_{2,14}^{l *}=0.00, \quad \alpha_{3,14}^{l *}=0.00 \\
& \lambda_{1}^{*}=0.02, \quad \lambda_{4}^{*}=0.15, \quad \lambda_{10}^{*}=0.04, \quad \lambda_{11}^{*}=0.79, \quad \lambda_{j}^{*}=0 \quad \forall j \neq 1,4,10,11 . \\
& \alpha_{1,12}^{u *}=2841.40, \alpha_{2,12}^{u *}=20.60, \alpha_{3,12}^{u *}=5335.33 \\
& \alpha_{u, 14}^{l *}=5793.10, \alpha_{2,14}^{u *}=0.00, \quad \alpha_{3,14}^{u *}=0.00 \\
& \lambda_{4}^{*}=0.18, \quad \lambda_{5}^{*}=0.04, \quad \lambda_{10}^{*}=0.03, \quad \lambda_{11}^{*}=0.70, \quad \lambda_{18}^{*}=0.05, \quad \lambda_{j}^{*}=0 \quad \forall j \neq 4,5,10,11,18 .
\end{aligned}
$$

Accordingly, to achieve the efficiency pre-defined target level $([0.96,1.00])$, the lower and upper bounds of the inherited input levels of the branch $B_{q}$ from merging units B12 and B14 are estimated and presented in Table 4.

The problem of merging entities based on the inverse DEA has been initially investigated by Gattoufi et al. [21]. Then, Zeinodin and Ghobadi [49] and Amin et al. [4] used MOP tools to estimate of the inherited inputs/outputs of the merged unit from merging units. In addition, the problem of merging units was studied with negative data and under inter-temporal dependence data by Amin and Al-Muharrami [1] and Zenodin and Ghobadi [50], respectively. Therefore, there is currently no inverse DEA based method for comparing the results of the IDEA based approach in this paper.

\section{CONCLusion}

Merging a group of DMUs to generate a new DMU is a method for cooperation of units to attain better performance. It is shown that the conventional inverse DEA based models could not be applicable in the presence of interval data. This paper studies the problem of merging units in the presence of interval data. By using the inverse DEA idea, sufficient conditions for identification of the inherited inputs/outputs of the merged DMU from merging DMUs to achieve a pre-defined efficiency target are extracted. In this way, Pareto solutions of MOLP problems have been utilized. In this paper, an insightful method is provided to obtain the minimum and maximum achievable lower and upper bounds of the efficiency interval of the merged unit. These minimum and maximum values could be considered as thresholds for the merger approval if these values are satisfactory. The applicability of the proposed models for target adjustment of the merged unit is demonstrated through a banking sector example. Nevertheless, any part including the homogeneous units with the merging ability could be considered as an issue for the proposed method in this paper.

In this paper, the IDEA approach is employed to study the merging DMUs problem when the merged unit (DMUq) is within the current PPS. Therefore, the following items could be considered as further research directions:

(i) Proposing a FDEA approach based solution for this problem.

(ii) Investigation of the problem of merging units when the merged unit is not within the current PPS. 


\section{Appendix A.}

Theorem A.1. If the following assumptions hold:

(i) The merged unit (DMUq) is within the current PPS;

(ii) $\Delta^{l}=\left(\lambda^{*}, \alpha_{i j}^{l *}: \forall i \in I, \forall j \in \Lambda\right)$ is a Pareto solution to model (3.2);

(iii) $x_{i q}^{l}=\sum_{j \in \Lambda} \alpha_{i j}^{l *}$ for each $i \in I$.

Then, the obtained efficiency upper bound of $D M U_{q}$ is equal to $\bar{\theta}_{q}^{u}$.

Proof. To prove the theorem, we should show that the optimal value of the following model is $\bar{\theta}_{q}^{u}$.

$$
\begin{aligned}
& \min \theta_{q}^{u} \\
& \text { s.t. } \quad \sum_{j \in \Pi} \lambda_{j} x_{i j}^{l}+\lambda_{q} x_{i q}^{l} \leq \theta_{q}^{u} x_{i q}^{l}, \quad i=1,2, \ldots, m, \\
& \sum_{j \in \Pi} \lambda_{j} y_{r j}^{u}+\lambda_{q} y_{r q}^{u} \geq y_{r q}^{u}, \quad r=1,2, \ldots, s, \\
& \sum_{j \in \Pi} \lambda_{j}+\lambda_{q}=1 \\
& \lambda_{j} \geq 0, \quad \forall j \in \Pi \cup\{q\} .
\end{aligned}
$$

Since $\bar{\theta}_{q}^{u} \leq 1$ and $\Delta^{l}$ is a feasible solution for model (3.2) (assumption (ii)), we have

$$
\begin{array}{ll}
\sum_{j \in \Pi} \lambda_{j}^{*} x_{i j}^{l} \leq \bar{\theta}_{q}^{u} \sum_{j \in \Lambda} \alpha_{i j}^{l *}=\bar{\theta}_{q}^{u} x_{i q}^{l} \leq x_{i q}^{l}, & \forall i \in I, \\
\sum_{j \in \Pi} \lambda_{j}^{*} y_{r j}^{u} \geq y_{r q}^{u}, & \forall r \in O, \\
0 \leq \alpha_{i j}^{l *} \leq x_{i j}^{l}, & \forall i \in I, \forall j \in \Lambda, \\
\sum_{j \in \Pi} \lambda_{j}^{*}=1 . &
\end{array}
$$

According to equations (A.2), (A.3), and (A.5), it is obvious that $\left(\bar{\lambda}=\left(\lambda_{j}^{*}, \forall j \in \Pi ; \lambda_{q}=0\right), \theta_{q}^{u}=\bar{\theta}_{q}^{u}\right)$ is a feasible solution to problem (A.1). Therefore, the optimal value of model (A.1) is less than or equal $\bar{\theta}_{q}^{u}$.

By contradiction assume that $\Phi=\left(\lambda_{j}^{* *}, \forall j \in \Pi ; \lambda_{q}^{* *}, \theta_{q}^{u * *}\right)$ be an optimal solution to model (A.1) in which $\theta_{q}^{u * *}<\bar{\theta}_{q}^{u} \leq 1$. The inequality (A.2) will be used in problem (A.1), the following result is obtained:

$$
\begin{aligned}
\theta_{q}^{u * *} x_{i q}^{l} \geq & \sum_{j \in \Pi} \lambda_{j}^{* *} x_{i j}^{l}+\lambda_{q}^{* *} x_{i q}^{l} \geq \sum_{j \in \Pi} \lambda_{j}^{* *} x_{i j}^{l}+\lambda_{q}^{* *}\left(\sum_{j \in \Pi} \lambda_{j}^{*} x_{i j}^{l}\right), \\
& \sum_{j \in \Pi}\left(\lambda_{j}^{* *}+\lambda_{q}^{* *} \lambda_{j}^{*}\right) x_{i j}^{l} \leq \theta_{q}^{u * *} x_{i q}^{l}, \quad \forall i \in I
\end{aligned}
$$

For each $j \in \Pi$, define $\bar{\lambda}_{j}=\lambda_{j}^{* *}+\lambda_{q}^{* *} \lambda_{j}^{*}$. By equation (A.5) and feasibility $\Phi$ to model (A.1), we have $\bar{\lambda}_{j} \geq 0$ $(\forall j \in \Pi)$ and

$$
\sum_{j \in \Pi} \bar{\lambda}_{j}=\sum_{j \in \Pi}\left(\lambda_{j}^{* *}+\lambda_{q}^{* *} \lambda_{j}^{*}\right)=\sum_{j \in \Pi} \lambda_{j}^{* *}+\lambda_{q}^{* *} \sum_{j \in \Pi} \lambda_{j}^{*}=1 .
$$


By (A.6) and $\theta_{q}^{u * *}<\bar{\theta}_{q}^{u}$ (contradiction assume), we have

$$
\sum_{j \in \Pi} \bar{\lambda}_{j} x_{i j}^{l} \leq \theta_{q}^{u * *} x_{i q}^{l}<\bar{\theta}_{q}^{u} x_{i q}^{l}, \quad \forall i \in I
$$

Similarly, considering equation (A.3) and problem (A.1), we get

$$
\sum_{j \in \Pi} \bar{\lambda}_{j} y_{r j}^{u} \geq y_{r q}^{u}, \quad \forall r \in O
$$

There exists at least one $p \in I$ and at least one $k \in \Pi$ such that $\alpha_{p k}^{l *}>0$, because $x_{i q}^{l} \neq 0$. Then, there exists positive scalar $\mu>0$, such that $\alpha_{p k}^{l *}-\mu \geq 0$ and

$$
\sum_{j \in \Pi} \bar{\lambda}_{j} x_{p j}^{l} \leq \bar{\theta}_{q}^{u}\left(\left(\alpha_{p k}^{l *}-\mu\right)+\sum_{j \in \Lambda-\{k\}} \alpha_{p j}^{l *}\right) .
$$

Now, define

$$
\bar{\alpha}_{i j}^{l}= \begin{cases}\alpha_{i j}^{l *}-\mu & \text { if } i=p, j=k, \\ \alpha_{i j}^{l *} & \text { otherwise. }\end{cases}
$$

By (A.8), (A.10), and (A.11), we have

$$
\begin{array}{ll}
\sum_{j \in \Pi} \bar{\lambda}_{j} x_{i j}^{l} \leq \bar{\theta}_{q}^{u} \sum_{j \in \Lambda} \bar{\alpha}_{i j}^{l}, \quad \forall i \in I, \\
0 \leq \bar{\alpha}_{i j}^{l} \leq x_{i j}^{l}, & \forall i \in I .
\end{array}
$$

According to (A.7), (A.9), (A.12), and (A.13), $\left(\bar{\lambda}, \bar{\alpha}_{i j}^{l}: \forall i \in I, \forall j \in \Lambda\right)$ is a feasible solution to problem (3.2), in which $\left(\bar{\alpha}_{i j}^{l} ; \forall i \in I, \forall j \in \Lambda\right) \leq\left(\bar{\alpha}_{i j}^{l *} ; \forall i \in I, \forall j \in \Lambda\right)$ and $\left(\bar{\alpha}_{i j}^{l} ; \forall i \in I, \forall j \in \Lambda\right) \neq\left(\bar{\alpha}_{i j}^{l *} ; \forall i \in I, \forall j \in \Lambda\right)$. This contradicts the assumption (ii) that $\Delta^{l}$ is a Pareto solution to problem (3.2). Therefore, $\theta_{q}^{u * *}=\bar{\theta}_{q}^{u}$ and the proof is completed.

\section{Appendix B.}

Theorem B.1. If the following assumptions hold:

(i) The merged unit $(D M U q)$ is within the current PPS;

(ii) $\Delta^{u}=\left(\lambda^{*}, \alpha_{i j}^{u *}: \forall i \in I, \forall j \in \Lambda\right)$ is a Pareto solution to MOLP (3.4) in which $\alpha_{i j}^{u *} \neq \bar{\alpha}_{i j}^{l}$ for some $i \in I$ and $j \in \Lambda$;

(iii) $x_{i q}^{u}=\sum_{j \in \Lambda} \alpha_{i j}^{u *}$ for each $i \in I$.

Then, the obtained efficiency lower bound of $D M U_{q}$ is equal to $\bar{\theta}_{q}^{l}$.

Proof. To prove the theorem, we consider the following LP problem:

$$
\begin{array}{ll}
\min & \theta_{q}^{l} \\
\text { s.t. } & \sum_{j \in \Pi} \lambda_{j} x_{i j}^{l}+\lambda_{q} x_{i q}^{l} \leq \theta_{q}^{l} x_{i q}^{u}, \quad i=1,2, \ldots, m,
\end{array}
$$




$$
\begin{array}{ll}
\sum_{j \in \Pi} \lambda_{j} y_{r j}^{u}+\lambda_{q} y_{r q}^{u} \geq y_{r q}^{l}, & r=1,2, \ldots, s, \\
\sum_{j \in \Pi} \lambda_{j}+\lambda_{q}=1, & \\
\lambda_{j} \geq 0, & \forall j \in \Pi \cup\{q\} .
\end{array}
$$

We should show that the optimal value of the above model is $\bar{\theta}_{q}^{l}$.

Since the efficiency frontiers are identical before and after merging (assumption (i)), then in each optimal solution of the above model we have $\lambda_{q}^{*}=0$. Accordingly, model (B.1) can be converted to the following model:

$$
\begin{array}{lll}
\min & \theta_{q}^{l} \\
\text { s.t. } & \sum_{j \in \Pi} \lambda_{j} x_{i j}^{l} \leq \theta_{q}^{l} x_{i q}^{u}, \quad & \\
& \sum_{j \in \Pi} \lambda_{j} y_{r j}^{u} \geq y_{r q}^{l}, & \\
& \sum_{j \in \Pi} \lambda_{j}=1,2, \ldots, m, \\
& \lambda_{j} \geq 0, & \\
& \forall j \in \Pi .
\end{array}
$$

Feasibility of $\Delta^{u}$ for model (3.4), implies that $\left(\lambda^{*}, \theta_{q}^{l}=\bar{\theta}_{q}^{l}\right)$ is a feasible solution to problem (B.2). Therefore, the optimal value of model (B.2) is less than or equal $\bar{\theta}_{q}^{l}$.

By contradiction assume that $\Phi=\left(\lambda^{* *}, \theta_{q}^{l * *}\right)$ be an optimal solution to model (B.2) in which $\theta_{q}^{l * *}<\bar{\theta}_{q}^{l} \leq 1$. Feasibility of $\Phi$ for model (B.2) and $\theta_{q}^{l * *}<\bar{\theta}_{q}^{l}$ (contradiction assume), implies

$$
\begin{array}{ll}
\sum_{j \in \Pi} \lambda_{j}^{* *} x_{i j}^{l} \leq \theta_{q}^{l * *} x_{i q}^{u}<\bar{\theta}_{q}^{l} x_{i q}^{u}=\bar{\theta}_{q}^{l} \sum_{j \in \Lambda} \alpha_{i j}^{u *}, & \forall i \in I, \\
\sum_{j \in \Pi} \lambda_{j}^{* *} y_{r j}^{u} \geq y_{r q}^{l}, & \forall r \in O, \\
\sum_{j \in \Pi} \lambda_{j}^{* *}=1 . &
\end{array}
$$

If assumption (ii) holds, then there exists at least one $p \in I$ and at least one $k \in \Lambda$ such that $\bar{\alpha}_{p k}^{l}<\alpha_{p k}^{u *}$. Therefore, there exists positive scalar $\mu>0$, such that $\bar{\alpha}_{p k}^{l} \leq \alpha_{p k}^{u *}-\mu$ and

$$
\sum_{j \in \Pi} \lambda_{j}^{* *} x_{p j}^{l} \leq \bar{\theta}_{q}^{l}\left(\left(\alpha_{p k}^{u *}-\mu\right)+\sum_{j \in \Lambda-\{k\}} \alpha_{p j}^{u *}\right) .
$$

Now, define

$$
\bar{\alpha}_{i j}^{u}= \begin{cases}\alpha_{i j}^{u *}-\mu & \text { if } \quad i=p, j=k, \\ \alpha_{i j}^{u *} & \text { otherwise. }\end{cases}
$$

By (B.3), (B.6), and (B.7), we have

$$
\sum_{j \in \Pi} \lambda_{j}^{* *} x_{i j}^{l} \leq \bar{\theta}_{q}^{l} \sum_{j \in \Lambda} \bar{\alpha}_{i j}^{u}, \quad \forall i \in I,
$$




$$
\bar{\alpha}_{i j}^{l} \leq \bar{\alpha}_{i j}^{u} \leq x_{i j}^{u}, \quad \forall i \in I .
$$

According to (B.4), (B.5), (B.8), and (B.9), $\left(\lambda^{* *}, \bar{\alpha}_{i j}^{u}: \forall i \in I, \forall j \in \Lambda\right)$ is a feasible solution to problem (3.4), in which $\left(\bar{\alpha}_{i j}^{u} ; \forall i \in I, \forall j \in \Lambda\right) \leq\left(\alpha_{i j}^{u *} ; \forall i \in I, \forall j \in \Lambda\right)$ and $\left(\bar{\alpha}_{i j}^{u} ; \forall i \in I, \forall j \in \Lambda\right) \neq\left(\alpha_{i j}^{u *} ; \forall i \in I, \forall j \in \Lambda\right)$. This contradicts the assumption (ii) that $\Delta^{u}$ is a Pareto solution to MOLP (3.4). Accordingly, $\theta_{q}^{l * *}=\bar{\theta}_{q}^{l}$ and the proof is completed.

\section{Appendix C.}

Theorem C.1. If the following assumptions hold:

(i) The merged unit (DMUq) is within the current PPS;

(ii) $\Delta^{u}=\left(\lambda^{*}, \beta_{r j}^{u *}: \forall r \in O, \forall j \in \Lambda\right)$ is a weak Pareto solution to problem (3.6);

(iii) $y_{r q}^{u}=\sum_{j \in \Lambda} \beta_{r j}^{u *}$ for each $r \in O$.

Then, the obtained efficiency lower bound of $D M U_{q}$ is equal to $\bar{\varphi}_{q}^{l}$.

Proof. To prove the theorem, we should show that the optimal value of the following problem is $\bar{\varphi}_{q}^{l}$.

$$
\begin{aligned}
& \max \varphi_{q}^{l} \\
& \text { s.t. } \quad \sum_{j \in \Pi} \lambda_{j} x_{i j}^{l}+\lambda_{q} x_{i q}^{l} \leq x_{i q}^{l}, \quad i=1,2, \ldots, m, \\
& \sum_{j \in \Pi} \lambda_{j} y_{r j}^{u}+\lambda_{q} y_{r q}^{u} \geq \varphi_{q}^{l} y_{r q}^{u}, \quad r=1,2, \ldots, s, \\
& \sum_{j \in \Pi} \lambda_{j}+\lambda_{q}=1, \\
& \lambda_{j} \geq 0, \quad \forall j \in \Pi \cup\{q\} .
\end{aligned}
$$

Since $\Delta^{u}$ is a feasible solution for model (3.6) (assumption (ii)) and $\bar{\varphi}_{q}^{l} \geq 1$, we get

$$
\begin{array}{ll}
\sum_{j \in \Pi} \lambda_{j}^{*} x_{i j}^{l} \leq x_{i q}^{l}, & \forall i \in I, \\
\sum_{j \in \Pi} \lambda_{j}^{*} y_{r j}^{u} \geq \bar{\varphi}_{q}^{l} \sum_{j \in \Lambda} \beta_{r j}^{u *}=\bar{\varphi}_{q}^{l} y_{r q}^{u} \geq y_{r q}^{u}, & \forall r \in O, \\
\beta_{r j}^{u *} \geq y_{r j}^{u}, & \forall r \in O, \forall j \in \Lambda, \\
\sum_{j \in \Pi} \lambda_{j}^{*}=1 . &
\end{array}
$$

According to equations (C.2), (C.3), and (C.5), it is obvious that $\left(\bar{\lambda}=\left(\lambda_{j}^{*}, j \in \Pi ; \lambda_{q}=0\right), \varphi_{q}^{l}=\bar{\varphi}_{q}^{l}\right)$ is a feasible solution to problem (C.1). Therefore, the optimal value of model (C.1) is more than or equal $\bar{\varphi}_{q}^{l}$.

By contradiction assume that $\Phi=\left(\lambda_{j}^{* *}, j \in \Pi ; \lambda_{q}^{* *}, \varphi_{q}^{l * *}\right)$ be an optimal solution to model (C.1) in which $\varphi_{q}^{l * *}>\bar{\varphi}_{q}^{l} \geq 1$. The inequality (C.3) will be used in problem (C.1), the following result is obtained:

$$
\varphi_{q}^{l * *} y_{r q}^{u} \leq \sum_{j \in \Pi} \lambda_{j}^{* *} y_{r j}^{u}+\lambda_{q}^{* *} y_{r q}^{u} \leq \sum_{j \in \Pi} \lambda_{j}^{* *} y_{r j}^{u}+\lambda_{q}^{* *}\left(\sum_{j \in \Pi} \lambda_{j}^{*} y_{r j}^{u}\right),
$$




$$
\sum_{j \in \Pi}\left(\lambda_{j}^{* *}+\lambda_{q}^{* *} \lambda_{j}^{*}\right) y_{r j}^{u} \geq \varphi_{q}^{l * *} y_{r q}^{u}, \quad \forall r \in I
$$

For each $j \in \Pi$, define $\bar{\lambda}_{j}=\lambda_{j}^{* *}+\lambda_{q}^{* *} \lambda_{j}^{*}$. By equation (C.5) and feasibility $\Phi$ to model (C.1), we have $\bar{\lambda}_{j} \geq 0$ $(\forall j \in \Pi)$ and

$$
\sum_{j \in \Pi} \bar{\lambda}_{j}=\sum_{j \in \Pi}\left(\lambda_{j}^{* *}+\lambda_{q}^{* *} \lambda_{j}^{*}\right)=\sum_{j \in \Pi} \lambda_{j}^{* *}+\lambda_{q}^{* *} \sum_{j \in \Pi} \lambda_{j}^{*}=1 .
$$

Similarly, considering equation (C.2) and problem (C.1), we have

$$
\sum_{j \in \Pi} \bar{\lambda}_{j} x_{i j}^{l} \leq x_{i q}^{l}, \quad \forall i \in I .
$$

By (C.6) and $\varphi_{q}^{l * *}>\bar{\varphi}_{q}^{l}$ (contradiction assume), we have

$$
\sum_{j \in \Pi} \bar{\lambda}_{j} y_{r j}^{u} \geq \varphi_{q}^{l * *} y_{r q}^{u}>\bar{\varphi}_{q}^{l} y_{r q}^{u}=\bar{\varphi}_{q}^{l} \sum_{j \in \Lambda} \beta_{r j}^{u *}, \quad \forall r \in O
$$

Then, there exists scaler positive $\mu>0$, such that

$$
\sum_{j \in \Pi} \bar{\lambda}_{j} y_{r j}^{u} \geq \bar{\varphi}_{q}^{l} \sum_{j \in \Lambda}\left(\beta_{r j}^{u *}+\mu\right)=\bar{\varphi}_{q}^{l} \sum_{j \in \Lambda} \bar{\beta}_{r j}^{u}, \quad \forall r \in O
$$

According to (C.7), (C.8), and (C.10), $\left(\bar{\lambda}, \bar{\beta}_{r j}^{u}: \forall r \in O, \forall j \in \Lambda\right)$ is a feasible solution to problem (3.6), in which $\left(\bar{\beta}_{r j}^{u} ; \forall r \in O, \forall j \in \Lambda\right)>\left(\beta_{r j}^{u *} ; \forall r \in O, \forall j \in \Lambda\right)$. This contradicts the assumption (ii) that $\Delta^{u}$ is a weak Pareto solution to MOLP (3.6). Then, $\varphi_{q}^{l * *}=\bar{\varphi}_{q}^{l}$ and the proof is completed.

\section{Appendix D.}

Theorem D.1. If the following assumptions hold:

(i) The merged unit (DMUq) is within the current PPS;

(ii) $\Delta^{l}=\left(\lambda^{*}, \beta_{r j}^{l *}: \forall r \in O, \forall j \in \Lambda\right)$ is a Pareto solution to model (3.8) in which $\bar{\beta}_{r j}^{u} \neq \beta_{r j}^{l *}$;

(iii) $y_{r q}^{l}=\sum_{j \in \Lambda} \beta_{r j}^{l *}$ for each $r \in O$.

Then, the obtained efficiency upper bound of $D M U_{q}$ is equal to $\bar{\varphi}_{q}^{u}$.

Proof. To prove the theorem, we consider the following LP model:

$$
\begin{array}{lll}
\max & \varphi_{q}^{u} \\
\text { s.t. } & \sum_{j \in \Pi} \lambda_{j} x_{i j}^{l}+\lambda_{q} x_{i q}^{l} \leq x_{i q}^{u}, \quad & \\
& \sum_{j \in \Pi} \lambda_{j} y_{r j}^{u}+\lambda_{q} y_{r q}^{u} \geq \varphi_{q}^{u} y_{r q}^{l}, & \\
& \sum_{j \in \Pi} \lambda_{j}+\lambda_{q}=1,2, \ldots, m, \\
& \lambda_{j} \geq 0, & \\
& \forall j \in \Pi \cup\{q\} .
\end{array}
$$

we should show that the optimal value of the above model is $\bar{\varphi}_{q}^{u}$. 
Since the efficiency frontiers are identical before and after merging (assumption (i)), then in each optimal solution of the above model we have $\lambda_{q}^{*}=0$. Accordingly, model (D.1) can be converted to the following model:

$$
\begin{array}{lll}
\max & \varphi_{q}^{u} \\
\text { s.t. } & \sum_{j \in \Pi} \lambda_{j} x_{i j}^{l} \leq x_{i q}^{u}, \quad & \\
& \sum_{j \in \Pi} \lambda_{j} y_{r j}^{u} \geq \varphi_{q}^{u} y_{r q}^{l}, & r=1,2, \ldots, m, \\
& \sum_{j \in \Pi} \lambda_{j}=1, & \\
& \lambda_{j} \geq 0, & \forall j \in \Pi .
\end{array}
$$

Feasibility of $\Delta^{l}$ for model (3.8), implies that $\left(\lambda^{*}, \varphi_{q}^{u}=\bar{\varphi}_{q}^{u}\right)$ is a feasible solution to problem (D.2). Therefore, the optimal value of model (D.2) is more than or equal $\bar{\varphi}_{q}^{u}$.

By contradiction assume that $\Phi=\left(\lambda^{* *}, \varphi_{q}^{u * *}\right)$ be an optimal solution to model (D.2) in which $\varphi_{q}^{u * *}>\bar{\varphi}_{q}^{u} \geq 1$. Feasibility of $\Phi$ for model (D.2) and $\varphi_{q}^{u * *}>\bar{\varphi}_{q}^{u} \geq 1$ (contradiction assume), implies

$$
\begin{array}{ll}
\sum_{j \in \Pi} \lambda_{j}^{* *} x_{i j}^{l} \leq x_{i q}^{l}, & \forall i \in I, \\
\sum_{j \in \Pi} \lambda_{j}^{* *} y_{r j}^{u} \geq \varphi_{q}^{u * *} y_{r q}^{l}>\bar{\varphi}_{q}^{l} y_{r q}^{l}=\bar{\varphi}_{q}^{l} \sum_{j \in \Lambda} \beta_{r j}^{l *}, & \forall r \in O, \\
\sum_{j \in \Pi} \lambda_{j}^{* *}=1 . &
\end{array}
$$

If assumption (ii) holds, then there exists at least one $p \in O$ and at least one $k \in \Lambda$ such that $\beta_{p k}^{l *}<\bar{\beta}_{p k}^{u}$. Therefore, there exists posetive scalar $\mu>0$, such that $\beta_{p k}^{l *}+\mu \leq \bar{\beta}_{p k}^{u}$ and

$$
\sum_{j \in \Pi} \lambda_{j}^{* *} y_{p j}^{u} \geq \bar{\varphi}_{q}^{l}\left(\left(\beta_{p k}^{l *}+\mu\right)+\sum_{j \in \Lambda-\{k\}} \beta_{p j}^{l *}\right) .
$$

Now, define

$$
\bar{\beta}_{r j}^{l}= \begin{cases}\beta_{r j}^{l *}+\mu & \text { if } \quad r=p, j=k, \\ \beta_{r i j}^{l *} & \text { otherwise. }\end{cases}
$$

By (D.4), (D.6), and (D.7), we have

$$
\begin{array}{ll}
\sum_{j \in \Pi} \lambda_{j}^{* *} y_{r j}^{u} \geq \bar{\varphi}_{q}^{l} \sum_{j \in \Lambda} \bar{\beta}_{r j}^{l}, & \forall r \in O, \\
\bar{\beta}_{r j}^{u} \geq \bar{\beta}_{r j}^{l} \geq y_{r j}^{l}, & \forall r \in O .
\end{array}
$$

According to (D.3), (D.5), (D.8), and (D.9), $\left(\lambda^{* *}, \bar{\beta}_{r j}^{l}: \forall r \in O, \forall j \in \Lambda\right.$ ) is a feasible solution to problem (3.8), in which $\left(\bar{\beta}_{r j}^{l}: \forall r \in O, \forall j \in \Lambda\right) \geq\left(\beta_{r j}^{u *} ; \forall r \in O, \forall j \in \Lambda\right)$ and $\left(\bar{\beta}_{r j}^{l}: \forall r \in O, \forall j \in \Lambda\right) \neq\left(\beta_{r j}^{u *} ; \forall r \in O, \forall j \in \Lambda\right)$. This contradicts the assumption (ii) that $\Delta^{l}$ is a Pareto solution to problem (3.8). Therefore, $\varphi_{q}^{u * *}=\bar{\varphi}_{q}^{u}$ and the proof is completed. 


\section{Appendix E.}

Tables E.1 and E.2.

TABLE E.1. The inputs of 20 bank branches.

\begin{tabular}{llll}
\hline \hline Branches & Payable interest & Personnel & Non-performing loans \\
\hline B01 & {$[5007.37,9613.37]$} & {$[36.29,36.86]$} & {$[87243,87243]$} \\
B02 & {$[2926.81,5961.55]$} & {$[18.8,20.16]$} & {$[9945,12120]$} \\
B03 & {$[8732.7,17752.5]$} & {$[25.74,27.17]$} & {$[47575,50013]$} \\
B04 & {$[945.93,1966.39]$} & {$[20.81,22.54]$} & {$[19292,19753]$} \\
B05 & {$[8487.07,17521.66]$} & {$[14.16,14.8]$} & {$[3428,3911]$} \\
B06 & {$[13759.35,27359.36]$} & {$[19.46,19.46]$} & {$[13929,15657]$} \\
B07 & {$[587.69,1205.47]$} & {$[27.29,27.48]$} & {$[27827,29005]$} \\
B08 & {$[4646.39,9559.61]$} & {$[24.52,25.07]$} & {$[9070,9983]$} \\
B09 & {$[1554.29,3427.89]$} & {$[20.47,21.59]$} & {$[412036,413902]$} \\
B10 & {$[17528.31,36297.54]$} & {$[14.84,15.05]$} & {$[8638,10229]$} \\
B11 & {$[2444.34,4955.78]$} & {$[20.42,20.54]$} & {$[500,937]$} \\
B12 & {$[7303.27,14178.11]$} & {$[22.87,23.19]$} & {$[16148,21353]$} \\
B13 & {$[9852.15,19742.89]$} & {$[18.47,21.83]$} & {$[17163,17290]$} \\
B14 & {$[4540.75,9312.24]$} & {$[22.83,23.96]$} & {$[17918,17964]$} \\
B15 & {$[3039.58,6304.01]$} & {$[39.32,39.86]$} & {$[51582,55136]$} \\
B16 & {$[6585.81,13453.58]$} & {$[25.57,26.52]$} & {$[20975,23992]$} \\
B17 & {$[4209.18,8603.79]$} & {$[27.59,27.95]$} & {$[41960,43103]$} \\
B18 & {$[1015.52,2037.82]$} & {$[13.63,13.93]$} & {$[18641,19354]$} \\
B19 & {$[5800.38,11875.39]$} & {$[27.12,27.26]$} & {$[19500,19569]$} \\
B20 & {$[1445.68,2922.15]$} & {$[28.96,28.96]$} & {$[31700,32061]$} \\
\hline
\end{tabular}

TABLE E.2. The outputs of 20 bank branches.

\begin{tabular}{llllll}
\hline \hline Branches & $\begin{array}{l}\text { The total sum } \\
\text { of four main } \\
\text { deposits }\end{array}$ & Other deposits & Loans granted & Received interest & Fee \\
& {$[2696995,3126798]$} & {$[263643,382545]$} & {$[1675519,1853365]$} & {$[108634.76,125740.28]$} & {$[965.97,6957.33]$} \\
\hline B01 & {$[340377,440355]$} & {$[95978,117659]$} & {$[377309,390203]$} & {$[32396.65,37836.56]$} & {$[304.67,749.4]$} \\
B02 & {$[1027546,1061260]$} & {$[37911,503089]$} & {$[1233548,1822028]$} & {$[96842.33,108080.01]$} & {$[2285.03,3174]$} \\
B03 & {$[1145235,1213541]$} & {$[229646,268460]$} & {$[468520,542101]$} & {$[32362.8,39273.37]$} & {$[207.98,510.93]$} \\
B04 & {$[390902,395241]$} & {$[4924,12136]$} & {$[129751,142873]$} & {$[12662.71,14165.44]$} & {$[63.32,92.3]$} \\
B05 & {$[988115,1087392]$} & {$[74133,111324]$} & {$[507502,574355]$} & {$[53591.3,72257.28]$} & {$[480.16,869.52]$} \\
B06 & {$[144906,165818]$} & {$[180530,180617]$} & {$[288513,323721]$} & {$[40507.97,45847.48]$} & {$[176.58,370.81]$} \\
B07 & {$[408163,416416]$} & {$[405396,486431]$} & {$[1044221,1071812]$} & {$[56260.09,73948.09]$} & {$[4654.71,5882.53]$} \\
B08 & {$[335070,410427]$} & {$[337971,449336]$} & {$[1584722,1802942]$} & {$[176436.81,189006.12]$} & {$[560.26,2506.67]$} \\
B09 & {$[700842,768593]$} & {$[14378,15192]$} & {$[2290745,2573512]$} & {$[662725.21,791463.08]$} & {$[58.89,86.86]$} \\
B10 & {$[641680,696338]$} & {$[114183,241081]$} & {$[1579961,2285079]$} & {$[17527.58,20773.91]$} & {$[1070.81,2283.08]$} \\
B11 & {$[453170,481943]$} & {$[27196,29553]$} & {$[245726,275717]$} & {$[35757.83,42790.14]$} & {$[375.07,559.85]$} \\
B12 & {$[553167,574989]$} & {$[21298,23043]$} & {$[425886,431815]$} & {$[45652.24,50255.75]$} & {$[438.43,836.82]$} \\
B13 & {$[309670,342598]$} & {$[20168,26172]$} & {$[124188,126930]$} & {$[8143.79,11948.04]$} & {$[936.62,1468.45]$} \\
B14 & {$[286149,317186]$} & {$[149183,270708]$} & {$[787959,810088]$} & {$[106798.63,111962.3]$} & {$[1203.79,4335.24]$} \\
B15 & {$[321435,347848]$} & {$[66169,80453]$} & {$[360880,379488]$} & {$[89971.47,165524.22]$} & {$[200.36,399.8]$} \\
B16 & {$[618105,835839]$} & {$[244250,404579]$} & {$[9136507,9136507]$} & {$[33036.79,41826.51]$} & {$[2781.24,4555.42]$} \\
B17 & {$[248125,320974]$} & {$[3063,6330]$} & {$[26687,29173]$} & {$[9525.6,10877.78]$} & {$[240.04,274.7]$} \\
B18 & {$[640890,679916]$} & {$[490508,684372]$} & {$[2946797,3985900]$} & {$[66097.16,95329.87]$} & {$[961.56,1914.25]$} \\
B19 & {$[119948,120208]$} & {$[14943,17495]$} & {$[297674,308012]$} & {$[21991.53,27934.19]$} & {$[282.73,471.22]$} \\
B20 & & & & &
\end{tabular}


Acknowledgements. The author would like to thank Professor Nelson Maculan (the editor of the journal) and anonymous reviewers for their insightful and constructive comments and suggestions.

\section{REFERENCES}

[1] G.R. Amin and S. Al-Muharrami, A new inverse data envelopment analysis model for mergers with negative data. IMA J. Manage. Math. 29 (2018) 137-149.

[2] G.R. Amin and A. Oukil, Flexible target setting in mergers using inverse data envelopment analysis. Int. J. Oper. Res. 35 (2019) 301-317.

[3] G.R. Amin, A. Emrouznejad and S. Gattoufi, Modelling generalized firms' restructuring using inverse DEA. J. Prod. Anal. 48 (2017) 51-61.

[4] G.R. Amin, S. Al-Muharrami and M. Toloo, A combined goal programming and inverse DEA method for target setting in mergers. Expert Syst. App. 115 (2019) 412-417.

[5] X. Bai, J. Zeng and Y. Chiu, Pre-evaluating efficiency gains from potential mergers and acquisitions based on the resampling DEA approach: evidence from China's railway sector. Trans. Policy 76 (2019) 46-56.

[6] C. Bernad, L. Fuentelsaz and J. Gmez, The effect of mergers and acquisitions on productivity: an empirical application to Spanish banking. Omega 38 (2010) 283-293.

[7] P. Bogetoft and D.X. Wang, Estimating the potential gains from mergers. J. Prod. Anal. 23 (2005) 145-171.

[8] A. Charnes, W.W. Cooper and E. Rhodes, Measuring the efficiency of decision making units. Eur. J. Oper. Res. 2 (1978) $429-444$.

[9] W.D. Cook and L.M. Seiford, Data envelopment analysis (DEA)-Thirty years on. Eur. J. Oper. Res. 192 (2009) 1-17.

[10] W.W. Cooper, K.S. Park and G. Yu, IDEA and AR-IDEA: models for dealing with imprecise data in DEA. Manage. Sci. 45 (1999) 597-607.

[11] W.W. Cooper, L.M. Seiford and K. Tone, Data Envelopment Analysis: A Comprehensive Text With Models, Applications, References and DEA-Solver Software, Second edition. Springer US, New York, NY (2007).

[12] D. Despotis and Y. Smirlis, Data envelopment analysis with imprecise data. Eur. J. Oper. Res. 140 (2002) $24-36$.

[13] L. Dong Joon, Inverse DEA with frontier changes for new product target setting. Eur. J. Oper. Res. 254 (2016) $510-516$.

[14] M. Ehrgott, Multicriteria Optimization. Springer, Berlin-Heidelberg (2005).

[15] A. Emrouznejad and G. Yang, A survey and analysis of the first 40 years of scholarly literature in DEA: 1978-2016. J. Soc. Econ. Plan. Sci. 61 (2018) 4-8.

[16] A. Emrouznejad, B. Parker and G. Tavares, Evaluation of research in efficiency and productivity: a survey and analysis of the first 30 years of scholarly literature in DEA. J. Socio Econ. Plan. Sci. 42 (2008) 151-157.

[17] A. Emrouznejad, M. Rostamy-Malkhalifeh, A. Hatami-Marbini, M. Tavana and N. Aghayi, An overall profit Malmquist productivity index with fuzzy and interval data. Math. Comput. Model. 54 (2011) 2827-2838.

[18] A. Emrouznejad, M. Rostamy-Malkhalifeh, A. Hatami-Marbini and M. Tavana, General and multiplicative non-parametric corporate performance models with interval ratio data. Math. Comput. Model. 36 (2012) 5506-5514.

[19] A. Emrouznejad, G.-L. Yang and G.R. Amin, A novel inverse DEA model with application to allocate the $\mathrm{CO}_{2}$ emissions quota to different regions in Chinese manufacturing industries. J. Oper. Res. Soc. 70 (2018) 1-12.

[20] T. Entani, Y. Maeda and H. Tanaka, Dual models of interval DEA and its extension to interval data. Eur. J. Oper. Res. 136 (2002) 32-45.

[21] S. Gattoufi, G.R. Amin and A. Emrouznejad, A new inverse DEA method for merging banks. IMA J. Manage. Math. 25 (2014) 73-87.

[22] S. Ghobadi, Inputs and outputs estimation in inverse DEA. Iran. J. Optim. 9 (2017) 119-129.

[23] S. Ghobadi, Inverse DEA using enhanced Russell measure in the presence of fuzzy data. Int. J. Ind. Math. 10 (2018) 1-16.

[24] S. Ghobadi, A generalized DEA model for inputs (outputs) estimation under inter-temporal dependence. RAIRO: OR 53 (2019) 1791-1805.

[25] S. Ghobadi and S. Jahangiri, Optimal allocation of resources using the ideal-solutions. J. New Res. Math. 5 (2019) 121-134.

[26] S. Ghobadi, G.R. Jahanshahloo, F. Hoseinzadeh Lotfi and M. Rostami-Malkhalifeh, Dynamic inverse DEA in the presence of fuzzy data. Adv. Environ. Biol. 8 (2014) 139-151.

[27] S. Ghobadi, G.R. Jahanshahloo, F. Hoseinzadeh Lotfi and M. Rostami-Malkhalifeh, Efficiency measure under inter-temporal dependence. Int. J. Technol. Decis. Making 17 (2018) 657-675.

[28] A. Hadi-Vencheh, A.A. Foroughi and M. Soleimani-Damaneh, A DEA model for resource allocation. Econ. Model. 25 (2008) 983-993.

[29] G.R. Jahanshahloo, F.H. Lotfi and M. Moradi, Sensitivity and stability analysis in DEA with interval data. Appl. Math. Comput. 156 (2004) 463-477.

[30] G.R. Jahanshahloo, F.H. Lotfi, N. Shoja, G. Tohidi and S. Razavyan, Sensitivity of efficiency classifications in the inverse DEA models. Appl. Math. Comput. 169 (2005) 905-916.

[31] G.R. Jahanshahloo, F. Hoseinzadeh Lotfi, M. Rostami-Malkhalifeh and M. Ahadzadeh Namin, A generalized model for data envelopment analysis with interval data. Appl. Math. Model. 33 (2009) 3237-3244.

[32] G.R. Jahanshahloo, F. Hoseinzadeh Lotfi, M. Rostami-malkhalifeh and S. Ghobadi, Using enhanced Russell model to solve inverse data envelopment analysis problems. Sci. World J. 2014 (2014) 1-10. 
[33] G.R. Jahanshahloo, M. Soleimani-damaneh and S. Ghobadi, Inverse DEA under inter-temporal dependence using multipleobjective programming. Eur. J. Oper. Res. 240 (2015) 447-456.

[34] C. Kao, Interval efficiency measures in data envelopment analysis with imprecise data. Eur. J. Oper. Res. 174 (2006) 10871099.

[35] H. Leleu, J. Moises and V. Valdmanis, Optimal productive size of hospitals intensive care units. Int. J. Prod. Econ. 136 (2012) 297-305.

[36] F. Li, L. Liang, L. Yongjum and A. Emrouznejad, An alternative approach to decompose the potential gains from mergers. J. Oper. Res. Soc. 69 (2018) 1793-1802.

[37] H.T. Lin, An efficiency-driven approach for setting revenue target. Decis. Support Syst. 49 (2010) 311-317.

[38] H.H. Liu, T.Y. Chen and L.Y. Pai, The influence of merger and acquisition activities on corporate performance in the Taiwanese telecommunications industry. Serv. Ind. J. 27 (2007) 1041-1051.

[39] P. Molyneux, K. Schaeck and T. MiZhou, Too systemically important to fail in banking - evidence from bank mergers and acquisitions. J. Int. Money Finance 49 (2014) 258-282.

[40] V. Moonesian, S. Jahangiri and S. Ghobadi, Efficiency and super-efficiency under inter-temporal dependence. RAIRO:OR 54 (2020) 1385-1400.

[41] J. Motis, Mergers and acquisitions motives, Working Papers 0730. University of Crete, Department of Economics (2007).

[42] J.K. Sengupta, A fuzzy systems approach in data envelopment analysis. Comput. Math. App. 24 (1992) $259-266$.

[43] X. Shi, Y. Li, A. Emrouznejad, J. Xie and L. Liang, Estimation of potential gains from bank mergers: a novel two-stage cost efficiency DEA model. J. Oper. Res. Soc. 9 (2017) 1045-1055.

[44] A.H. Shokouhi, A. Hatami-Marbini, M. Tavana and S. Saati, A robust optimization approach for imprecise data envelopment analysis. Comput. Ind. Eng. 59 (2010) 387-397.

[45] Y. Wang, R. Greatbanks and J. Yang, Interval efficiency assessment using data envelopment analysis. Fuzzy Sets Syst. 153 (2005) 347-370.

[46] P. Wanke, A. Maredza and R. Gupta, Merger and acquisitions in South African banking: a network DEA model. Res. Int. Bus. Finance 41 (2017) 362-376.

[47] J.A. Weber and U.M. Dholakia, Including marketing synergy in acquisition analysis: a step-wise approach. Ind. Market. Manage. 29 (2000) 157-177.

[48] M. Wegener and G.R. Amin, Minimizing greenhouse gas emissions using inverse DEA with an application in oil and gas. Expert Syst. App. 122 (2019) 369-375.

[49] E. Zeinodin and S. Ghobadi, Merging DMUs based on of the idea inverse DEA. Iran. J. Optim. 11 (2019) 77-84.

[50] E. Zeinodin and S. Ghobadi, Merging decision-making units under inter-temporal dependence. IMA J. Manage. Math. 31 (2020) $139-166$.

[51] X. Zhang and J. Cui, A project evaluation system in the state economic information system of China: an operation research practice in public sectore. Int. Trans. Oper. 6 (1999) 441-452. 